\title{
Sodium channel distribution in the apical dendrites of pyramidal cells vary in the hindbrain of Apteronotus leptorhynchus.
}

\author{
Sree Indrani Motipally \\ West Virginia University, srmotipally@mix.wvu.edu
}

Follow this and additional works at: https://researchrepository.wvu.edu/etd

Part of the Integrative Biology Commons, Molecular and Cellular Neuroscience Commons, and the Other Neuroscience and Neurobiology Commons

\section{Recommended Citation}

Motipally, Sree Indrani, "Sodium channel distribution in the apical dendrites of pyramidal cells vary in the hindbrain of Apteronotus leptorhynchus." (2019). Graduate Theses, Dissertations, and Problem Reports. 3773.

https://researchrepository.wvu.edu/etd/3773

This Thesis is protected by copyright and/or related rights. It has been brought to you by the The Research Repository @ WVU with permission from the rights-holder(s). You are free to use this Thesis in any way that is permitted by the copyright and related rights legislation that applies to your use. For other uses you must obtain permission from the rights-holder(s) directly, unless additional rights are indicated by a Creative Commons license in the record and/ or on the work itself. This Thesis has been accepted for inclusion in WVU Graduate Theses, Dissertations, and Problem Reports collection by an authorized administrator of The Research Repository @ WVU. For more information, please contact researchrepository@mail.wvu.edu. 
2019

Sodium channel distribution in the apical dendrites of pyramidal cells vary in the hindbrain of Apteronotus leptorhynchus.

Sree Indrani Motipally

Follow this and additional works at: https:// researchrepository.wvu.edu/etd

Part of the Integrative Biology Commons, Molecular and Cellular Neuroscience Commons, and the Other Neuroscience and Neurobiology Commons 
Sodium channel distribution in the apical dendrites of pyramidal cells vary in the hindbrain of Apteronotus leptorhynchus.

\author{
Sree Indrani Motipally
}

Thesis submitted to the Eberly College of Arts and Sciences at West Virginia University in partial fulfillment of the requirements for the degree of

\title{
Master of Science in Biology
}

Gary Marsat, Ph.D., Chair

Andrew M. Dacks, Ph.D.

Sarah M. Farris, Ph.D.

Department of Biology Morgantown, WV, 2019

Keywords: Bursting, Voltage gated Sodium Channels

Sensory systems, Electroreception, Neural coding Copyright 2019 Sree Indrani Motipally 


\title{
ABSTRACT \\ Sodium channel distribution in the apical dendrites of pyramidal cells vary in the hindbrain of Apteronotus leptorhynchus.
}

\author{
Sree Indrani Motipally
}

Apteronotid weakly electric fish heavily rely on their electrosensory system for behaviors like spatial navigation, communication and prey capture. Since the behaviorally important information about their environment is contained in the spatial and temporal modulations of the electrosensory signal, efficient mechanisms to process this information with great fidelity are of the utmost importance. Efficient sensory processing often involves having multiple parallel processing streams so that each stream can specialize to treat signals with different properties. This strategy requires the response properties and neural dynamic to be adjusted in each pathway to implement different neural coding strategies. One of the neural coding strategies employed by the primary electrosensory area is to use bursts of spikes in response to specific temporal features of the signal - a coding strategy described as feature-extraction. Burst generation relies on dendritic voltagegated sodium channels (Nav channels) expressed on pyramidal cell apical dendrites to support the active backpropagation of somatic spikes and the generation of depolarizing after-potentials. The presence and role of these Nav channels is well documented but variation in their expression across processing stream has not been investigated. Considering that many of the other ion channels expressed in these cells show differences across pathways, we hypothesize that Nav expression varies across the 3 electrosensory lateral line segments (lateral, centro-lateral and centro-medial segments; LS, CLS, CMS respectively) representing different processing streams. We used immunocytochemistry and confocal imaging of hindbrain slices to quantify differences in density and distribution of Nav channels in the apical dendrites of pyramidal cells. The dendritic Nav channel distribution follows a mediolateral gradient with lateral segment of the ELL exhibiting the highest density. We also found that dendritic Nav channel densities remain fairly constant across the proximal and distal locations of the apical dendrites across maps with CMS showing slightly higher Nav density in distal regions. We argue that the differences we observed may contribute to shaping the response properties and the specialization of each processing stream thereby contributing to the efficiency of the sensory system. 


\section{ACKNOWLEDGMENTS}

This work would not have been possible without the financial support from West Virginia University. I would like to express my sincere gratitude to my advisor Dr. Gary Marsat for his continuous support, encouragement and patience during my Masters research. I could not have asked for a more kind and understanding advisor. I would also like to thank my thesis committee members Dr. Andrew Dacks for his support, guidance and insightful comments; and Dr. Sarah Farris for being very supportive and encouraging throughout.

My sincere thanks also go to Dr. Saravanan Kolandaivelu for offering me his valuable time and resources for troubleshooting my experiments.

I thank my fellow lab mates Kate Allen and Daniel Williamson for creating a supportive, happy and fun lab environment, and for all the fun we had outside the lab.

Last but not the least, I would like to thank my family and friends, especially my mother Nagaveni Motipally, who has always been encouraging, in everything I pursued. 


\section{TABLE OF CONTENTS}

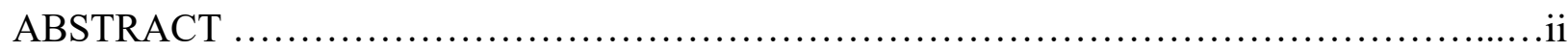

ACKNOWLEDGMENTS ..................................................................

TABLE OF CONTENTS ….................................................................

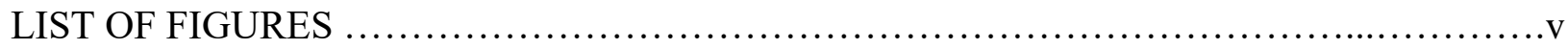

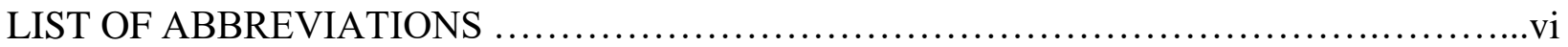

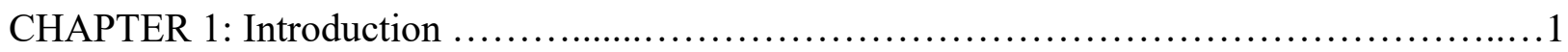

I. Intrinsic conductances shape firing dynamics.....................................

II. Bursts and bursting dynamics .............................................................

III. Electrosensory System in Apteronotus leptorhynchus...............................2

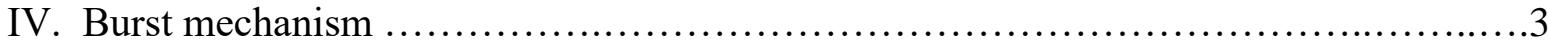

$\mathrm{V}$. Role of bursts in electric fish neural coding ...................................... 4

VI. Known differences across the ELL.................................................

CHAPTER 2: Differences in sodium channel densities across segments in the apical dendrites of

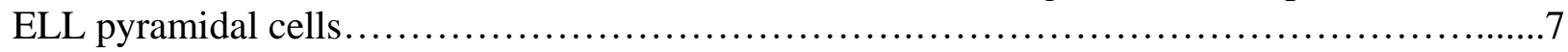

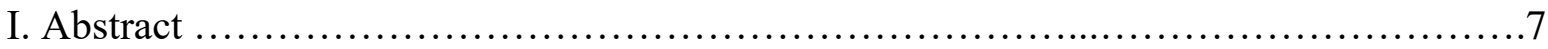

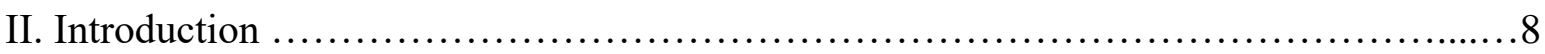

III. Materials and Methods ..............................................................

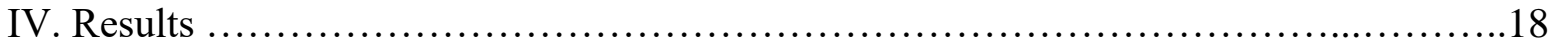

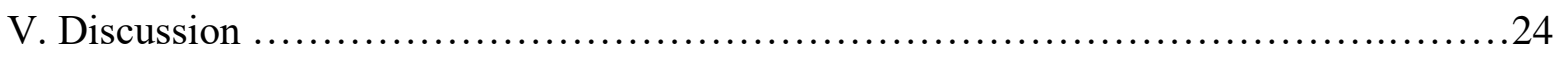

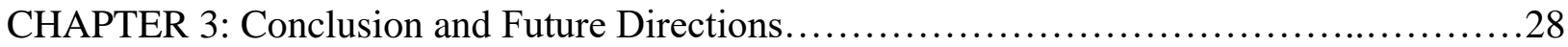

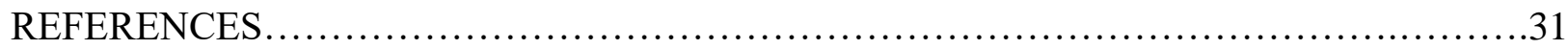




\section{LIST OF FIGURES}

Figure 1. Pyramidal cell and backpropagation ........................................ 11

Figure 2. : Scanning and localization procedure. ................................... 13

Figure 3. Immunohistochemistry revealed a punctate expression of Nav channels on the apical

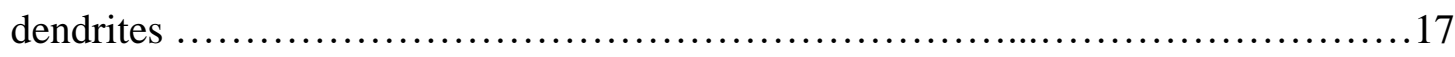

Figure 4. Examples of Nav-Map 2 expression in the ELL. .............................20

Figure 5. Schematic representation of the entire dataset................................ 21

Figure 6. Nav channel expression density differs across maps..........................23

Figure 7. Inter-spike intervals (ISI) histogram for burst-spikes during spontaneous activity....24 


\section{LIST OF ABBREVIATIONS}

\begin{tabular}{|c|c|}
\hline ELL & Electrosensory lateral line lobe \\
\hline EOD & Electric organ discharge \\
\hline $\mathrm{PC}$ & Pyramidal cell \\
\hline PCL & Pyramidal cell layer \\
\hline VML & Ventral molecular layer \\
\hline DML & Dorsal molecular layer \\
\hline LS & Lateral segment \\
\hline CLS & Centro-lateral segment \\
\hline CMS & Centro-medial segment \\
\hline Nav & Voltage gated sodium channels \\
\hline MAP2 & Microtubule associated protein 2 \\
\hline SK & Small conductance calcium-activated potassium channel \\
\hline TTX & Tetrodotoxin \\
\hline $\mathrm{tSF}$ & Tractus stratum fibrosum \\
\hline DAP & Depolarizing after potential \\
\hline ISI & Interspike interval \\
\hline AHP & After-hyperpolarization \\
\hline $\mathrm{AM}$ & Amplitude modulation \\
\hline
\end{tabular}




\section{CHAPTER 1: INTRODUCTION}

\section{Intrinsic conductances shape firing dynamics}

Spike generation and spiking patterns are affected by many cellular parameters, such as morphology, subcellular distribution of ion channels and receptor channel composition, etc. Intrinsic properties of the neurons dictate to a large extent their functional dynamics. For example, cells can produce tonic or phasic responses depending on their intrinsic properties and variations in currents can generate different response (Llináis, 2014). Voltage gated sodium channels (Nav channels) are most famously involved in the generation of action potential in axons but many electrophysiological studies provide evidence for their localization on dendrites (Turner et al., 1991). Their ability to promote retrograde conduction of a spike from soma to dendrite (Stuart and Sakmann, 1994), can underlie a distinct mode of spiking response called bursting. In these bursting neurons, the dendritic Nav channels have been localized to a specific compartment of the dendritic tree proximal to the soma. Our goal here is to characterize possible variations in Nav channel expression as a way to understand how neural properties are adjusted across segments.

\section{Bursts and bursting dynamics}

Many studies indicate that bursts play an important role in brain function as they carry precise stimulus related information. Sensory bursts are best characterized in relay neurons of the mammalian thalamus and in hindbrain pyramidal cells (PCs) in the electric fish electrosensory system. Bursts have been shown to be functional units of information and carry more information when compared to single spikes (Cattaneo et al., 1981). Bursts more reliably signal the presence of specific features of the stimulus (Guido et al., 1992, 1995; Mukherjee and Kaplan, 1995) and hence showcase a special role in neural signaling: supporting the detection of behaviorally important features. Furthermore, a single burst is capable and sufficient to bring about LTP and LDP (Huerta and Lisman, 1995) whereas a single spike cannot. This, once again, stresses its unique

role in neural processing. The behavioral role of bursts differs in different systems while retaining a common feature-detection function. For example, high frequency bursting in the cricket auditory sensory neuron -AN2 reflects significant increases in the ultrasound amplitude and triggers avoidance steering to escape echolocating bats (Marsat and Pollack, 2006). The behavioral 
relevance of stimulus features encoded via bursts has also been demonstrated in the Lateral Geniculate nucleus (Lesica and Stanley, 2004) where they signal sharp edges in contrast. In this system, the cellular properties supporting bursting and the way it responds and encodes stimuli is well understood: the low threshold and nonlinear amplification of the burst mechanism results in a large cortical response to a stimulus which otherwise would be insignificant to trigger a response (Lesica et al., 2006).

\section{Electrosensory system in Apteronotus leptorhynchus}

Another system where burst mechanism (Doiron et al., 2002; Turner et al., 1994) and its role is well understood is the Electrosensory Lateral Line lobe (ELL) of weakly electric fish. Since it is our model system, we first introduce it in the next paragraphs.

The electrosensory system plays a crucial role in spatial navigation, prey capture and communication in electric fish. A specialized organ called the electric organ generates pulse or waveform electric organ discharge (EOD), which can be transiently modulated to produce chirps that help in communication. Apteronotus leptorhynchus has a quasi-sinusoidal EOD that ranges from 650 to $1000 \mathrm{~Hz}$, with males having relatively higher EOD frequencies than females (Bastian and Nguyenkim, 2001). Objects and conspecifics in the vicinity of the fish affect the strength of the EOD locally thus creating "electrical shadows" and changes in amplitude of the EOD on corresponding portions of the skin (Nelson and Maciver, 1999).

Electroreceptors distributed all over the fish's body carry out both passive and active electroreception. They are classified into 2 major classes. Ampullary receptors that are tuned to exogenous low frequency electric fields thereby mediating passive electroreception. Tuberous receptors that are tuned to fish's own EOD and implement active electroreception (Kawasaki, 2005). Tuberous receptors are further categorized into P- and T-units based on their response to EOD. T-units are time coding and carry phase information about EOD cycles. The more abundant P-units fire probabilistically in response to the EOD and their firing rate correlates with stimulus amplitude (Nelson et al., 1997). P-units and their targets constitute the largest component of the electrosensory system; since we will focus on this pathway we will simply refer to P-units as "receptors". 
Each receptor trifurcates terminating in three different segments of the ELL to form a distinct somatotopic map in each segment (Heiligenberg and Dye, 1982). Pyramidal cells (PCs) of the ELL project to higher brain regions namely, the nucleus praeminentialis (nP) and torus semicircularis (TS) in the midbrain. The 3 ELL segments of interest are named the centromedial (CMS), centrolateral (CLS) and lateral (LS) segments (Figure 1A).

The ELL is a laminar structure with distinct layers and several subtypes of PCs. The deepest layer of the ELL consists of electroreceptor afferents and their terminal boutons that synapse onto both PCs and interneurons. The main populations of output neurons in the ELL are PCs, which are divided into basilar (ON-cell) and non-basilar (OFF-cell) subtypes encoding increases or decreases in amplitude of the EOD (Maler, 1979) analogous to ON cells and OFF cells of the visual system. $\mathrm{ON}$ and OFF-cells are found adjacent to each other in a columnar organization, processing the sensory input from the same patch of skin. They are further classified into superficial, intermediate and deep PCs based on the dorso-ventral position of their soma in the ELL layers (Bastian and Courtright, 1991). The 3 maps of the ELL differ in several ways (see Krahe and Maler, 2014 for a recent review and paragraph below). For example, the receptive field of the PCs vary from smaller (CMS) to larger (LS). Frequency tuning, particularly in ON cells, is low-pass in the CMS and highpass in LS. Neuromodulation affects LS more heavily than CLS. These property differences tunes them adequately to process different types of signals: spatially diffuse communication signals in LS and small localized prey-like objects in CMS (Marsat et al., 2009; Metzner et al., 1998). Most variations observed follow a medio-lateral gradient with CLS having properties intermediate between CMS and LS.

\section{$\underline{\text { IV. Burst mechanism }}$}

Bursting follows a "ping-pong" mechanism in which spikes generated in the soma by a depolarizing current, propagate into the apical dendrites and activate dendritic Nav channels. These backpropagating dendritic sodium spikes cause the current to flow back electrotonically generating a depolarizing after potential (DAP) at the soma, which can bring the soma past

threshold again thus leading to production of another spike with a short ISI (Figure 1B). Somatic spikes are followed by both fast and slow afterhyperpolarizations (AHPs). Increase in DAP amplitude due to repetitive spike discharge decreases the ability of somatic AHPs to hyperpolarize 
the cell and thereby increases the frequency of spike discharge. The increased frequency of spike discharge triggers a high frequency doublet, which is followed by burst afterhyperpolarization (Lemon and Turner, 2000; Turner et al., 2002). High frequency doublets terminate bursts since dendritic backpropagation ceases when the somatic ISI falls within dendritic sodium channel refractory period (Fernandez et al., 2005). In vivo, the full burst cycle is often truncated leading to burst with more variable ISI structures and with typically less spikes (e.g. most in-vivo burst are 2 or 3 spikes long), but the reason for this difference is still unknown. Various models of this burst dynamic have established the role of the ionic and morphological aspects at play (Noonan et al., 2003).

\section{$\underline{\text { V. Role of bursts in electric fish neural coding }}$}

Bursting is a dynamic process during which neuronal activity alternates between quiescent and repetitive spiking states. It plays an important role in communication between neurons which is essential for pattern generations and synchronization(Izhikevich, 2006). ELL PCs generate spike bursts which were found to be always biased towards low AM frequency events whereas isolated spikes code high frequency stimuli. Previous studies showed that the frequency selectivity across ELL maps correlates with the properties of spike bursts in vitro (Shumway, 1989; Turner et al., 1996). Due to the bursting dynamic, bursts act as reliable feature detectors (Gabbiani et al., 1996; Lisman, 1997) and signal the presence of prey-like (CLS,CMS; Oswald et al., 2004) or chirp-like stimuli (LS; Marsat et al., 2009). However, bursts contribute less than single spikes to encode the detailed time course of the stimulus (Oswald et al., 2004). Burst coding varies across layers of the ELL due to the morphological differences of the PCs. Deep PCs have small apical dendrites and display a more tonic firing rate. They are very distinct from intermediate and superficial PCs because they project to a different part of a feedback loop-nP, rather than projecting upstream to the TS. Intermediate and superficial PCs also differ in their apical dendrite size and spiking pattern but with a less distinct division between the 2 sub-types. Despite these differences, the role of bursts in coding follows the same strategy in all of the PC subtypes. They signal the occurrence of specific stimulus features rather than encoding the detailed time-course of the signal.

Oswald et al, (2007) studied the relationship between the DAP size, burst structure (i.e. the number and intervals between bursts) and the information carried by bursts. Specifically, they 
show that interval between spikes in a burst are directly correlated with the slope and height of the peak in amplitude that triggered the burst. They demonstrated that burst ISIs should differ by a minimum of $2 \mathrm{~ms}$ to efficiently encode the differences in stimulus intensity. They used LIF-DAP model that reproduces the in-vitro spiking characteristics of ELL PCs to broadband inputs and mimics the interval coding. Their modeling study showed that the moderate size of DAP promotes coding maximal information about stimulus intensity by the ISIs. Furthermore, it shows that optimal DAP size is inversely related to the variance of the stimulus (Doiron et al., 2007). While these studies point to the relevance of DAP size in shaping neural coding, we do not know if this parameter is in fact adjusted across PC subtypes to influence how each category of neurons processes information.

\section{Known differences across the ELL}

Since the burst mechanism and DAP generation is so crucial to determining the exact nature of the neural code implemented by burst, any changes in response properties affecting this mechanism can alter the coding scheme used by the PCs. The ELL segments display several such differences that specializes each segment for different tasks. For example, PCs exhibit differences in their frequency tuning across maps (Mehaffey et al., 2008b). Frequency tuning of CLS and LS cells was observed to be dependent on the spatial extent of the stimulus whereas the tuning in CMS cells is independent of it. CMS ON-cells show low pass characteristics to both local and global stimulation whereas CLS ON-cells are low-pass filters for local but show high pass properties for global signals. Response characteristics of LS ON-cells depend on the type of stimulation. They display similar tuning properties as CMS ON-cells when stimulated with a sinusoidal AM, but show high frequency response for global random AM stimulus (Krahe et al., 2008). LS cells possess larger spatial receptive fields and show higher adaptation rate to step changes in amplitude when compared to other maps (Shumway, 1989). Map-specific frequency tuning is established in part by the variations in the intrinsic ion channels of ON and OFF-type PCs, where OFF-cells remain relatively constant across segments, but ON-cells differ in ion channel composition (Mehaffey et al., 2008a). Most notably, SK channels, which regulate the frequency selectivity and tuning, are highly expressed in LS cells when compared to CLS and CMS (Ellis et al., 2007b). The key role of dendritic Nav channels in burst generation leads us to wonder if their localization and 
density on apical dendrites varies between segments thereby contributing to differences in response properties observed between them. Immunocytochemical localization of sodium channels using $\mathrm{NaCh}$ immunolabel has shown the active regions of dendrites (i.e. dendritic Nav) to be localized in patches, with intervening regions of relatively passive membrane (Turner et al., 1994). The authors focused on the first $\sim 200 \mu \mathrm{m}$ of apical dendrites where backpropagating action potentials are observed, but they noted the presence of Nav channels higher in the apical dendrites. The role of these channels in supporting the backpropagation of somatic spikes was confirmed by dendritic TTX ejections (Turner et al., 1994) and modeling. They did not provide a quantitative description of the distribution of Nav channels but unpublished observation (Maler, personal communication) suggest potential differences across segments. This observation would be in line with the other numerous cellular, coding and functional differences across segments.

Based on the hypothesis that dendritic Nav channel distribution varies across segment, the goal of this thesis is to reveal how specialization of neurons across different sensory pathways can be achieved by adjusting the density in expression of a given channel. This thesis can thus serve to better understand the link between cellular/molecular properties and the neural basis of behavior. 


\title{
CHAPTER 2: DIFFERENCES IN SODIUM CHANNEL DENSITIES ACROSS SEGMENTS IN THE APICAL DENDRITES OF ELL PYRAMIDAL CELLS
}

\author{
A modified version of this article is published in the journal Frontiers in Neural Circuits: Motipally SI, \\ Allen KM, Williamson DK \& Marsat G. Differences in sodium channel densities in the apical dendrites of pyramidal \\ cells of the electrosensory lateral line lobe. Frontiers in Neural Circuits. In Press (Accepted April 2019). \\ Contributions: SIM designed the study, performed the immunocytochemistry and Western Blot, analyzed \\ the data and wrote the paper. KMA performed a GABA labelling experiment (not presented in this thesis). DKW \\ performed the neural recordings used for Fig 7. GM designed the study, analyzed the data and wrote the paper
}

\section{$\underline{\text { I. Abstract }}$}

Heterogeneity of neural properties within a given neural class is ubiquitous in the nervous system and permits different sub-classes of neurons to specialize for specific purposes. This principle has been thoroughly investigated in the hindbrain of the weakly electric fish $A$. leptorhynchus in the primary electrosensory area, the Electrosensory Lateral Line lobe (ELL). The ELL pyramidal cells that receive inputs from tuberous electroreceptors are organized in three maps. The properties of these ON-cells vary greatly across maps due to differences in connectivity, receptor expression, and ion channel composition. These ON-cells are a seminal example of bursting neurons and their bursting dynamic relies on the presence of voltage-gated $\mathrm{Na}^{+}$channels in the extensive apical dendrites of the superficial pyramidal cells. Other ion channels can affect burst generation, for e.g., SK channels cause hyperpolarizing after-potentials and their expression varies across ELL neurons, yet bursting propensity is similar across segments. We question whether the depolarizing mechanism that generates the bursts presents quantitative differences across segments that could counterbalance other differences having the opposite effect. Although their presence and role are established, the distribution and density of the apical dendrites' $\mathrm{Na}^{+}$ channels have not been quantified and compared across ELL maps. Therefore, we hypothesize that $\mathrm{Na}^{+}$channel density will vary across segment and we test this by quantifying their distribution in the apical dendrites of immunolabeled ELL sections. We found the $\mathrm{Na}^{+}$channels to be two-fold denser in the lateral map than in the centro-medial map, the centro-lateral map being intermediate.. Our results imply that this differential expression of voltage-gated $\mathrm{Na}^{+}$channel could counterbalance or interact with other aspects of neuronal physiology that vary across segments (e.g. SK channels). We argue that burst coding of sensory signals, and the way the network regulates bursting, should be influenced by these variations in $\mathrm{Na}^{+}$channel density. 


\section{Introduction}

Neurons possess a variety of ion channels and membrane proteins that shape their response properties, from the classical $\mathrm{Na}^{+}$and $\mathrm{K}^{+}$ion channels generating action potentials to G-protein coupled receptors (Duménieu et al., 2017). Heterogeneity in neuron's physiology can be understood through two complementary principles. One perspective stresses that a given neural output can result from various composition of channels and proteins. This principle was most obviously demonstrated in the stomatogastric ganglion of crab where an identical motor output pattern could be generated using network that differed widely in their channel composition (Prinz et al., 2004). This work highlighted that a change in one element of the neuron's physiology can be compensated by changes in another element.

The other "non-exclusive" principle is also a basic concept in neuroscience. Specialization of neurons for different purposes and for performing different computations often involves changing the composition of their membrane proteins (Hille, 2001). An example of this principle, central to the subject of our study, comes from neurons that possesses specific ionic conductances responsible for generating burst of spikes (Krahe and Gabbiani, 2004). The neuron's bursting dynamic could not be possible without these specific ion channels and their role in neural coding is thus changed by this bursting dynamic.

Bursting is observed in various sensory systems and typically fulfils the same function: signaling the occurrence of specific spatio-temporal patterns of inputs (Gabbiani et al., 1996; Kepecs et al., 2002). In the visual system, bursts signal edges and sharp contrasts (Lesica and Stanley, 2004), in the cricket auditory system they signal salient ultrasound pulses typical of insectivorous bats (Marsat and Pollack, 2006, 2012) and in the electrosensory system they signal prey-like peaks in signal amplitude or aggressive communication signals (Gabbiani et al., 1996; Oswald et al., 2004). The presence of the bursting mechanism is thus key in shaping the neuron's role in the sensory pathway. The present study focuses on this bursting property and investigates variations in the channels responsible for burst generation in the sensory system of weakly electric fish.

The electrosensory lateral line lobe (ELL) is the primary sensory area in the hindbrain of gymnotid weakly electric fish and the main output neurons, pyramidal cells (PCs), possess a well characterized bursting mechanism (Doiron et al., 2002; Turner et al., 1994). PCs, particularly the 
more superficial ones, have extensive apical dendrites dedicated to receiving feedback inputs but these dendrites also support the generation of bursts. These apical dendrites extend several hundred $\mu \mathrm{m}$ into the molecular layer (Figure 1A) dorsal to the pyramidal cell layer (PCL) (Bastian and Courtright, 1991). They contain TTX-sensitive voltage-gated sodium channels (Nav channels). When an action potential is initiated in the cell, the somatic potential backpropagates actively 200 $\mu \mathrm{m}$ up the apical dendrites due to the Nav conductance. Current from the backpropagating action potential then flows back down to the soma passively causing a depolarizing after-potential (DAP) after each spike (Figure 1B; Turner et al., 1994). In vitro and in models, this backpropagation mechanism can trigger a sequence of several spikes with increasingly shorter interspike intervals (ISIs). This stereotyped bursting dynamic, named ghostbursting (Doiron et al., 2002), might not unfold in the same way in vivo where bursts are typically truncated to be only a few spikes-long. Nevertheless, backpropagation and the DAP are an integral part of the bursting dynamic and thus shape burst-coding of sensory signals (Oswald et al., 2007).

Response properties of ELL PCs are shaped by a variety of other factors that vary across cell subtypes (Maler, 2007). PCs are classified based on their location in the ELL layer. Superficial and intermediate PCs have extensive apical dendrites while deep PCs have short apical dendrites and fulfill a different role in the circuit (Bastian et al., 2004; Bastian and Nguyenkim, 2001). PCs receive inputs from receptors either directly through an inverting interneuron leading their response patterns to be typical of ON-cells and OFF-cells respectively (Maler, 1979); both types have the same burst dynamic. The ELL is organized in several topographic maps. While the map of the medial segment is driven by ampullary electroreceptors, the centro-medial, centro-lateral and lateral segments (CMS, CLS and LS respectively) receive inputs from the tuberous receptors sensitive to the fish's self-generated electric signal (Kawasaki, 2005). In this study, we focus on the ELL maps responsible for processing this active electrosensory signal since it plays a key role in navigation, prey capture and communication.

PCs from the 3 maps vary widely in their response properties. These differences are due to variations in network connectivity, ion channels composition, expression of neuromodulator receptors and more (Ellis et al., 2008; Krahe and Maler, 2014; Maler, 2009). These differences in properties allow the specialization of the three maps for different purposes: whereas the more medial segment is well suited for the localization of small near-by objects such as prey, the more lateral segments might specialize for processing communication signals (Marsat et al., 2009; 
Metzner and Juranek, 1997). Despite these differences in properties and function, all segments display similar bursting proportions (Krahe et al., 2008). Although small differences in burst patterns have been noted, e.g. some variations were observed in the ISIs occurring within bursts across segments with LS showing shortest ISIs. (Mehaffey et al., 2008b; Metzner et al., 1998; Turner et al., 1996), it is unclear to what extent burst propensity varies across segments and no fundamental differences have been documented in burst coding (Krahe et al., 2008; Oswald et al., 2004).

Large differences, particularly in bursting rates, could have been expected across different segments given the known differences in conductances affecting bursting. SK channels, which generate a hyperpolarizing after-potential, vary in expression across PCs subtypes. SK2 channels are particularly prevalent in the LS where they oppose the DAP-based burst generation mechanism (Ellis et al., 2007a, 2008). Serotonin receptors are also expressed differently, with LS cells expressing more and when activated, serotonergic inputs can enhance bursting propensity (Deemyad et al., 2011, 2013; Johnston et al., 1990). It is thus clear that many factors interact to shape bursting and it is possible that similarities in burst coding across PC subtypes and segments happens despite differences in intrinsic configurations rather than because they have identical physiology.

Variation in one element central to the burst-generation mechanism has not been examined yet: Nav channel expression in the apical dendrites of PCs. Considering the differences related to the burst mechanism noted above, we hypothesize that variations in the expression of Nav channels will also be observed across segments. To test this hypothesis, we performed immunocytochemistry on ELL slice labelling Nav channels in the molecular layer of the 3 segments of the ELL. We observed Nav expression throughout the molecular layer and we show that it is denser in LS than CMS. We argue that this differential expression should have functional consequences on response properties but that it is hard to determine how it interacts with the many other differences seen across segments. 


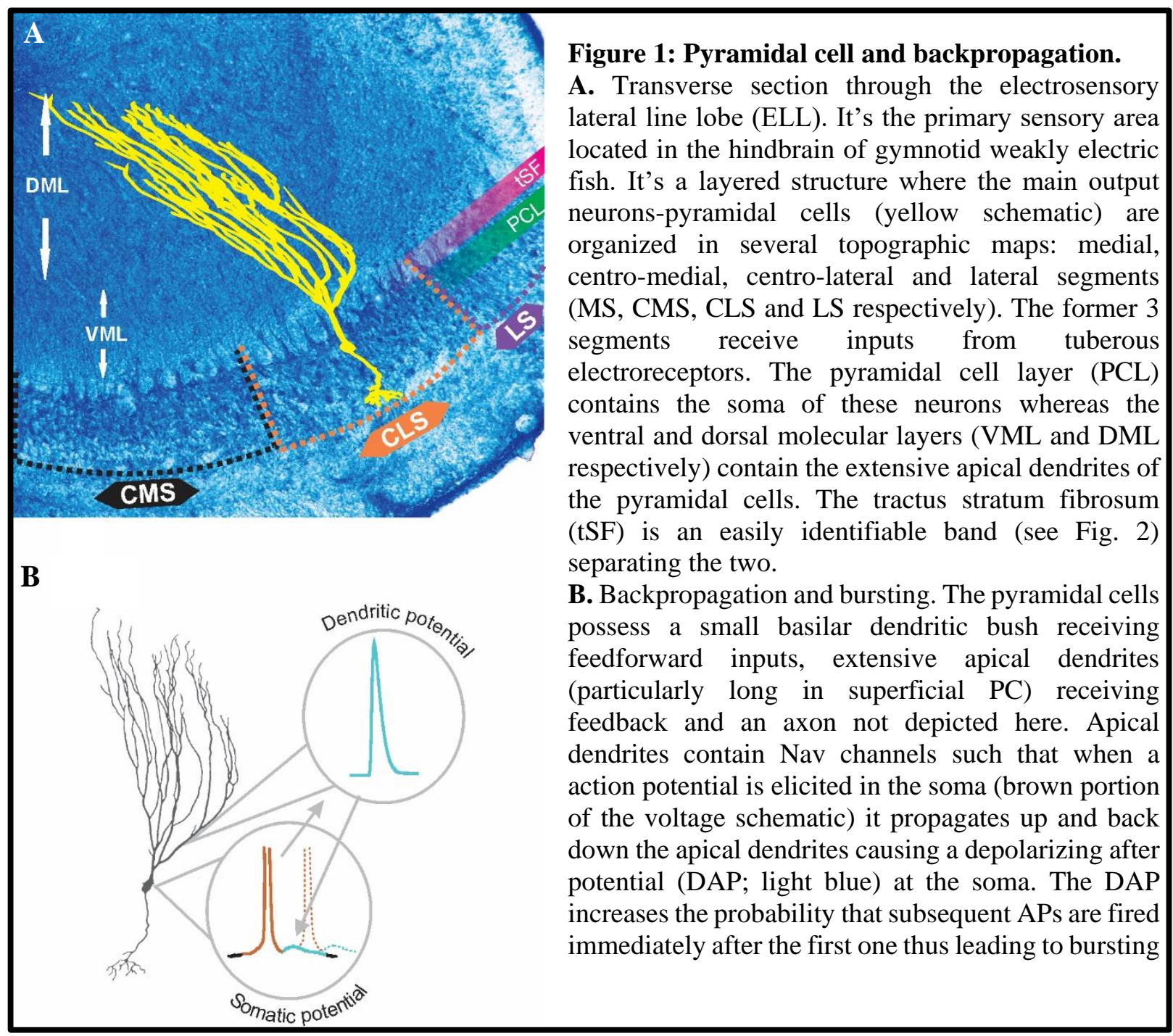

\section{Materials and methods}

\section{Apteronotus brain cryosection preparation}

Apteronotus leptorhynchus fish used for experiments were wild-caught and purchased from a tropical fish supplier (Segrest Farms, FL, USA). Fish were maintained in home tanks $(61 \times 30.5 \times 50.8 \mathrm{~cm})$ at $26-27^{\circ} \mathrm{C}, 250-300 \mu \mathrm{S}$ on inverted light cycles, fed ad libitum and were provided with environmental enrichment. Fish of either sex were anesthetized in tank water with MS-222 (3-amino benzoic acid ethyl ester) and respirated with oxygen bubbled MS-222 water during perfusion. All chemicals were obtained from Fisher scientific (Hampton, NH) unless 
otherwise noted. Heart was surgically exposed and intracardial perfusion was performed via the Conus arteriosus with $5 \mathrm{ml}$ of cold 0.9\% saline containing Heparin (\# 9041-08-1), NaNO2 (\# $\mathrm{S} 25560$ ) and $\mathrm{NaCl}$ (\# 7647-14-5) which is followed by perfusion with $40 \mathrm{~mL}$ of cold $4 \%$ paraformaldehyde (Electron Microscopy Sciences, \#RT-15714) in 1X-phosphate buffered saline (PBS), pH-7.3. Whole brains were surgically removed and post fixed in $4 \%$ paraformaldehyde (PFA) in 1X PBS for 4 hours at $4^{\circ} \mathrm{C}$ and were washed three times for 15 minutes each in $1 \mathrm{X}$ PBS at $4^{\circ} \mathrm{C}$. Brains were sequentially cryoprotected in $20 \%$ and $30 \%$ sucrose (\# S25590) in 1X-PBS, $\mathrm{pH}-7.3$ until they were completely saturated and later incubated in 1:1 mixture of $30 \%$ sucrose solution and optical cutting temperature (OCT) compound (Electron Microscopy Sciences, \#62550-01) for 1-2 hours before embedding in OCT. Dry-ice chilled 100\% ethanol was used to freeze the brain in OCT in a cryomold and the mold was incubated at $-80^{\circ} \mathrm{C}$ for $1-2$ hours before sectioning. 15-20 $\mu \mathrm{m}$ thick transverse brain sections were obtained using cryostat (Leica 1850) and the slides were stored at $4{ }^{\circ} \mathrm{C}$ for immediate processing or stored at $-20^{\circ} \mathrm{C}$ until use.

\section{Immunohistochemistry}

Brain sections were labelled for Nav in situ through the following procedure. Sections were washed 3 times with 1X-PBS, pH-7.3 for 5 minutes each and were blocked for 1 hour in 5\% normal goat serum (\# 005-000-121, Jackson Immuno Research) in PBSAT (1X PBS, 5mM sodium azide and $0.1 \%$ Triton $\mathrm{X}-100)$. Blocking was followed by 1-hour incubation with Anti-Pan Nav (Alomone labs, \# ASC003) (1:50) and purified Mouse Anti-MAP II (BD biosciences, \#556320) (1:400) primary antibodies in blocking buffer at room temperature. Later, brain sections were transferred to $4{ }^{\circ} \mathrm{C}$ for overnight incubation. Note that the MAP2 antibody used in the current study only stains the high molecular weight isoforms of MAP2 and does not recognize low molecular weight MAP2 isoforms or other microtubule proteins. In addition, MAP2 is mainly concentrated in the dendritic part of the nerve cells (Olesens, 1994), this might possibly explain the comparatively fainter MAP2 labelling observed in the cell bodies (Fig 3C). 


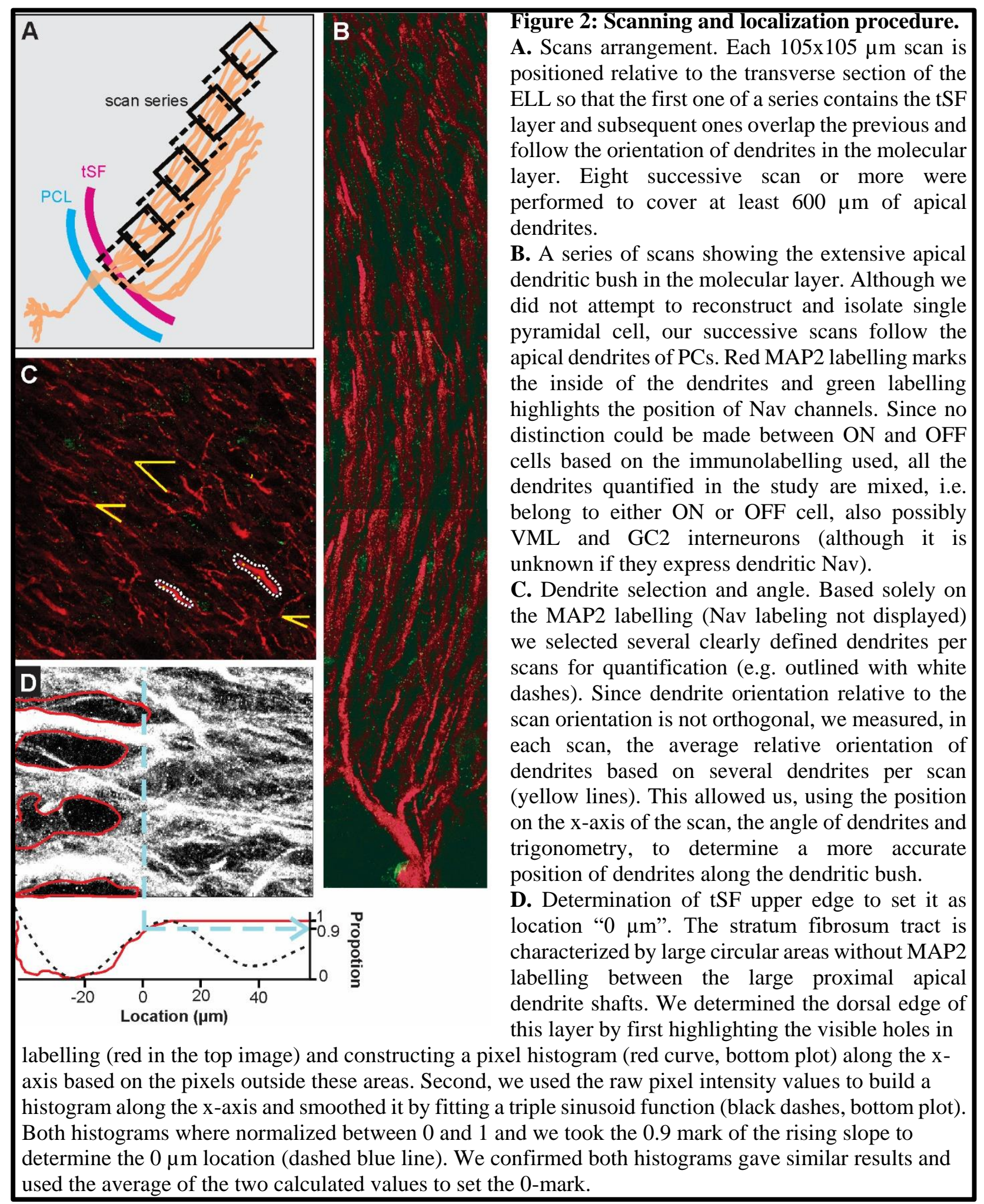


Sections were washed 4 times with 1X-PBST (1X PBS and 0.1\% Triton X-100), pH-7.3 for 15 minutes each and were incubated with Goat anti rabbit Alexa 488 (Life Technologies, \#A11008) (1:500) and Goat anti Mouse Alexa 546 (Thermofisher, \#A-11030) (1:500) secondary antibodies for 3 hours at room temperature in an enclosed moist chamber. Sections were washed 4 times with 1X-PBS, pH-7.3 for 15 minutes each and were mounted in Vectashield (Vector Laboratories, \# H-1000) and coverslipped. Selectivity of the labelling was confirmed with several controls: an absorption control where the Nav antibody is incubated with the immunogen (Figure 3G-IA), a control with no primary antibody (Figure 3J) and a quantification of Nav labelling in regions where no Nav channels are expected to be found. For this last control, we selected 27 different 10x10um areas in the gaps between dendrite in the tSF layer (where we do not expect Nav channels to be present) and compared the Nav labeling to 27 areas selected randomly on the same scans but in the middle of the dendritic arbors in the molecular layer. As expected, minimal staining was found (gaps in tSF: 0.009 puncta/ $\mu \mathrm{m}^{2}$; molecular layer: 0.1 puncta/ $\mu \mathrm{m}^{2}$; T-Test: $\mathrm{p}<10^{-}$ $\left.{ }^{6}\right)$ proving that puncta of labeling are not simply an artefact and randomly distributed on the tissue.

\section{Western blot verifying antibody specificity}

The specificity of polyclonal Anti Pan-Nav antibody was evaluated using western blot analysis (Figure 3F). Unperfused whole brain was surgically removed and frozen in liquid nitrogen and immediately homogenized with cold homogenization buffer containing $250 \mathrm{mM}$ Sucrose, 1mM EDTA, 10mM Tris HCL and protease inhibitor cocktail (\#4693132001, Sigma Aldrich), pH7.2, on pre-chilled mortar and pestle. Tissue sample was sonicated using two 10 second pulses with 30 second interval between each sonication. Sample was centrifuged at 500X g for 10 minutes to remove intact cells, nuclei and cell debris, and the supernatant was centrifuged at $100000 \mathrm{X} \mathrm{g}$ for $1 \mathrm{hr}$ at $4{ }^{\circ} \mathrm{C}$. Supernatant was discarded, and the pellet was resuspended in homogenization buffer and centrifuged at $100000 \mathrm{X}$ at $4{ }^{\circ} \mathrm{C}$ for 1 hour. Resultant supernatant was discarded and the pellet containing the membrane fractions was used to run western blot.

For western blot, 1X LDS (\#NP 0008, Thermofisher) was added to the protein samples and was heated for $10 \mathrm{~min}$ at $70^{\circ} \mathrm{C}$. $5 \mathrm{uL}$ of the sample was loaded into $4-6 \%$ polyacrylamide gel along with Precision Plus Protein ${ }^{\mathrm{TM}}$ Dual Color Standard (\#1610374S, Bio-Rad) and was run at $120 \mathrm{~V}$. Transfer was done at 100mA for $22 \mathrm{hrs}$ at $4{ }^{\circ} \mathrm{C}$ and the nitrocellulose membrane (\# 162-0094, BioRad) was blocked for 1 hour with 5\% BSA and 0.05\% NaN 3 in 1X TBST (1X TBS, 0.1\% Tween) 
under agitation. Primary antibody diluted in the blocking buffer (1:200) was applied to the membrane and incubated overnight at $4{ }^{\circ} \mathrm{C}$ under gentle agitation. After incubation, membrane was washed 3 times with $1 \mathrm{X}$ TBST for 15 minutes each. HRP conjugated anti-rabbit secondary antibody (\#A0545, Sigma) was applied at 1:10,000 dilution and agitated for 1 hour at room temperature, which was followed by three 15-minute washes with 1X TBST. ECL substrate (\#RPN2109, GE Healthcare) was added and the membrane was imaged on FluorChemQ system (Protein Simple).

\section{Nav density quantification}

Scans were obtained on FV-1000 Fluoview confocal microscope and minor brightness adjustments were made using Fluoview software. All of the scans were imaged using 60X oil immersion lens at $2 \mathrm{X}$ digital zoom. Scan size $\mathrm{X}^{*} \mathrm{Y}$ is set at $105.4^{*} 105.4 \mu \mathrm{m}$ with $\mathrm{Z}$ at $0.5 \mu \mathrm{m}$. Differences in the spatial distribution across the brain maps is assessed by quantitative image analysis using VAA-3D software. Image $\mathrm{J}$ software was used to perform image normalization. To measure the spatial distribution of dendritic Nav channels across different ELL maps (LS, CLS and CMS), the dorsal edge of the tSF layer is chosen as reference location $(0 \mu \mathrm{m})$. Scans covered the first 600 to $800 \mu \mathrm{m}$ of apical dendrites as they project dorsally through the VML and DML layers.

In each scan, 2-4 portions of dendrites with clear MAP2 labeling were chosen prior to looking at the Nav labelling to prevent biased choice of dendrite based on expected channel density. Nav labeling was quantified by manually marking each puncta identified on the chosen dendrites. XY coordinates of each puncta and dendrites were stored for analysis. We were conservative in assigning a punctum as belonging to a dendrite and the numbers presented in this paper should be viewed as a lower-bound estimate. Note that any bias in quantifying puncta within a segment is also inherent to the quantifications done across segments, and therefore, the differences observed in Nav density across the segments are unlikely to be affected by such biases.

To standardize the identification of the edge of the tSF layer (defined as location $0 \mu \mathrm{m}$ ) a Matlab (Mathworks, Natick, MA) program was created to plot the density profile of the gaps present in the tSF layer along with the pixel intensity profile of the scan (see Figure 2D and explanation in the legend). 
A series of slightly overlapping scans of the entire extent of each map were obtained (Figure 2A) and the bleached area from the overlapped portion as well as a local landmark was used to obtain the start point $\mathrm{x}$-coordinate of each subsequent scan of the map. This allowed us to determine the orthogonal distance of each scan, dendrite and puncta from the tSF edge. Since dendrites do not travel orthogonally to the tSF edge and the scan orientation (Figure $2 \mathrm{~B} \& \mathrm{C}$ ), we had to correct the distances using dendrites' angles. To do so, in each scan we selected randomly 5 clear portions of dendrites and measured their angle relative to the scan's frame (Figure 2C). Using the average angle of the selected dendrites in each scan and trigonometry, orthogonal distances could be converted into estimated distance (i.e. distances that take into account the general curvature of dendrites). This estimate is still an underestimation of actual distance along the dendritic tree since dendrites are often not completely straight over one scan, and also any z-plane curvature was ignored. Note that we sectioned the hindbrain in a "true-transverse" orientation minimizing the curvature of dendrites in the z-plane over the proximal apical dendrites. This underestimation does not affect our conclusions but should be noted. Data analysis was performed with Matlab (Mathworks, Naticks, MA) and statistics with JMP (SAS Institute Inc, Cary, NC).

\section{Burst ISI characterization}

Fish were prepared for in vivo electrophysiological recordings as described in Marsat et al, (2009) where recording and analysis methods are also described in detail. Briefly, superficial $\mathrm{ON}$ and OFF-cells of the three segments were targeted and spontaneous activity was recorded for 60 seconds. Recorded spike trains were binarized and burst identified by first constructing an ISI histogram of the entire spike train and identifying the upper interval limit characteristic of the cell's burst ISIs (see Marsat and Pollack, 2012 for more details). Identified bursts were then used to calculate their ISI distribution. 


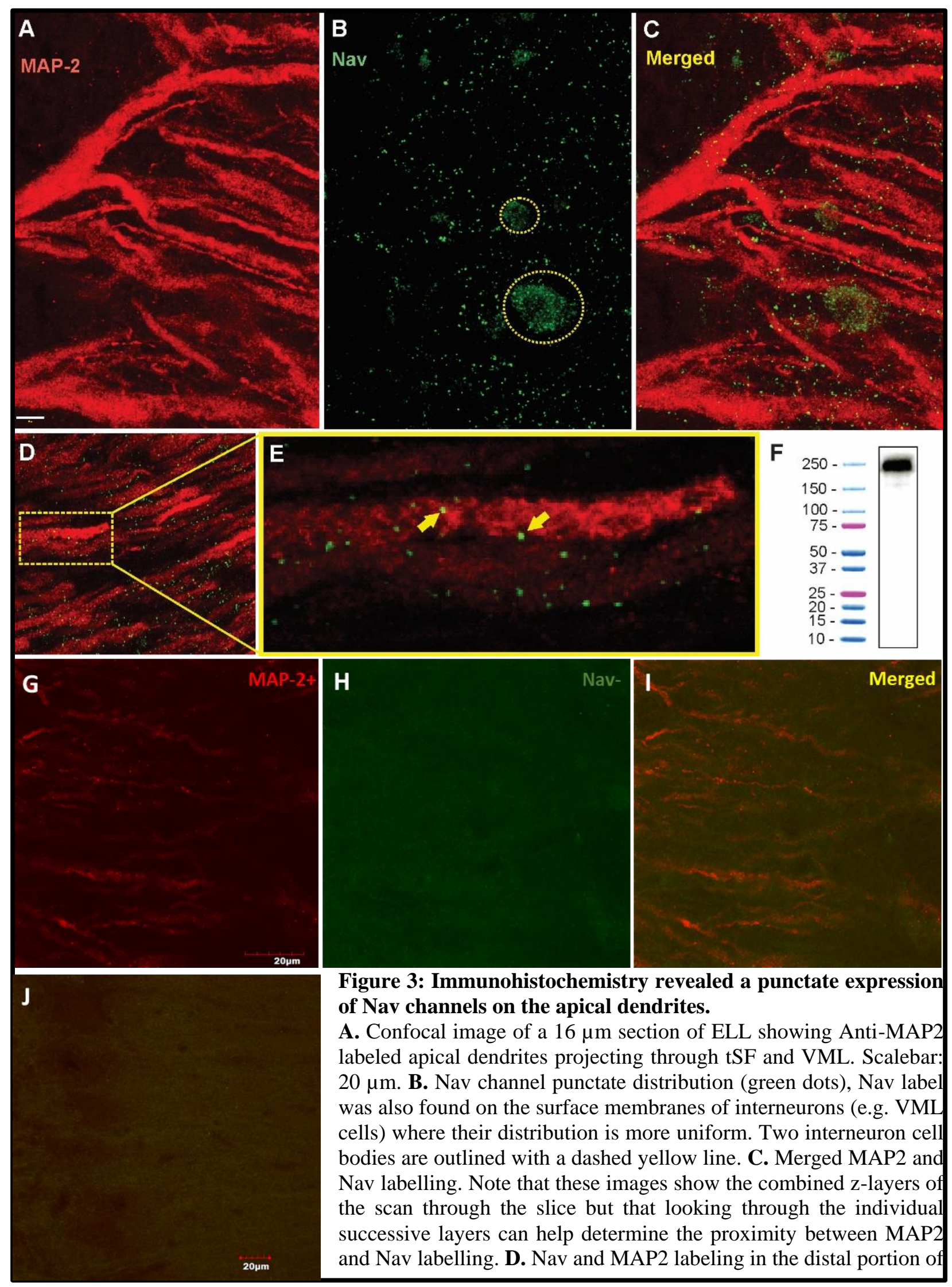


VML( $200 \mu \mathrm{m}$ from tSF-PCL boundary). E. Enlarged view of one dendrite in the image displayed in D selected for quantification of Nav channels. The proximity between the labelled Nav channels and the labeled MAP2 inside dendrites allows us to confirm that the channels where in the membrane of a pyramidal cell dendrite. Note that the Nav and MAP2 labelling does not need to overlap directly since MAP2 is located inside the dendrite and $\mathrm{Nav}$ channels are in the membrane. We thus expect some of the puncta to be slightly separated (in the x-y plane or the z plane) from the MAP2 labelling. F. Western blot analysis of brain tissue demonstrating the specificity of Anti-pan Nav antibody. The Western Blot of the tissue processed along with a protein ladder displayed a single band at $\sim 250 \mathrm{kDa}$. G,H,I. Absorption control: Confocal scan showing apical dendrites stained by Anti-MAP2(G) marker in the ELL-LS segment. Anti-pan Nav antibody was incubated with immunogen (peptide) in a 1:10 ratio overnight on a nutator at 4 degrees. Slide mounted ELL sections were incubated with pre-adsorbed Nav antibody along with Anti-MAP 2 primary antibody which was followed by incubation with their individual secondary antibodies. No Nav expression (H) was observed demonstrating the specificity of Nav antibody used. Scalebar - 20um. J. No primary antibody control: Anti-pan Nav and Anti MAP-II antibody used for labelling Nav and microtubules respectively were omitted from the IHC protocol as a control for non-specific binding of the secondary antibodies. Scalebar - 20um (1X Zoom). All scanning parameters were kept constant across treatment groups unless otherwise noted.

\section{$\underline{\text { IV. Results }}$}

To study the distribution of Nav channels along the apical dendrites of PCs we used a panNav antibody known to bind selectively to these channels in other tissues of this species (Ban et al., 2015). We confirmed the selectivity of the antibody in brain tissue by performing a Western blot (Figure 3F). To identify the position of the labeled channels relative to the dendrites, we also used a MAP2 antibody that densely labels the microtubules inside dendrites (Deng, 2005). By using thin brain slices $(15-20 \mu \mathrm{m})$ and high-resolution confocal scans we were able to precisely localize the labeled channels relative to PCs apical dendrites (Figure 3A-E). The pattern of expression of Nav channels in the dendrites followed a punctate fashion, as described previously (Turner et al., 1994), that is visibly different from the more uniform expression pattern in the soma of interneurons (Figure 3B) or in axons. Prior to visualizing the Nav labelling, we selected in each scan 2-4 portions of dendrites that are clearly delineated by the MAP2 labelling. We then visualized in 3D (moving through the z-plane with the scanning software) the position of each punctum in the vicinity of portion of dendrites selected for quantification. Since Nav channels are in the membrane of the dendrites and MAP2 proteins are inside the dendrite, Nav puncta were immediately adjacent (in the x-y plane or the z-plane) to the MAP2 labelling or overlapping.

Our dataset is based on images from a total of 15 brain slices ( 5 slices each from 3 fish). In each slice, all three segments were scanned and quantified thereby assuring that differences in immunolabeling clarity from slice to slice could not cause a bias in quantification across segments. 
Scans starting at the tSF layer and tiled to extend beyond $600 \mu \mathrm{m}$ into the molecular layer (Figure 4) allowed us to evaluate Nav expression as a function of segment and location along the ventraldorsal axis (i.e. proximal-distal to the soma). We localized and counted Nav puncta on 727 portions of dendrites representing over $21400 \mu \mathrm{m}$ of dendrites (Figure 5). We found a large dendrite-todendrite variability in puncta density with some long portions of dendrites containing no puncta and others being densely populated with puncta. This variability could be observed even between dendrites located side-by-side on the same scan precluding the possibility that variations in the immunolabeling process could account for this variability. 

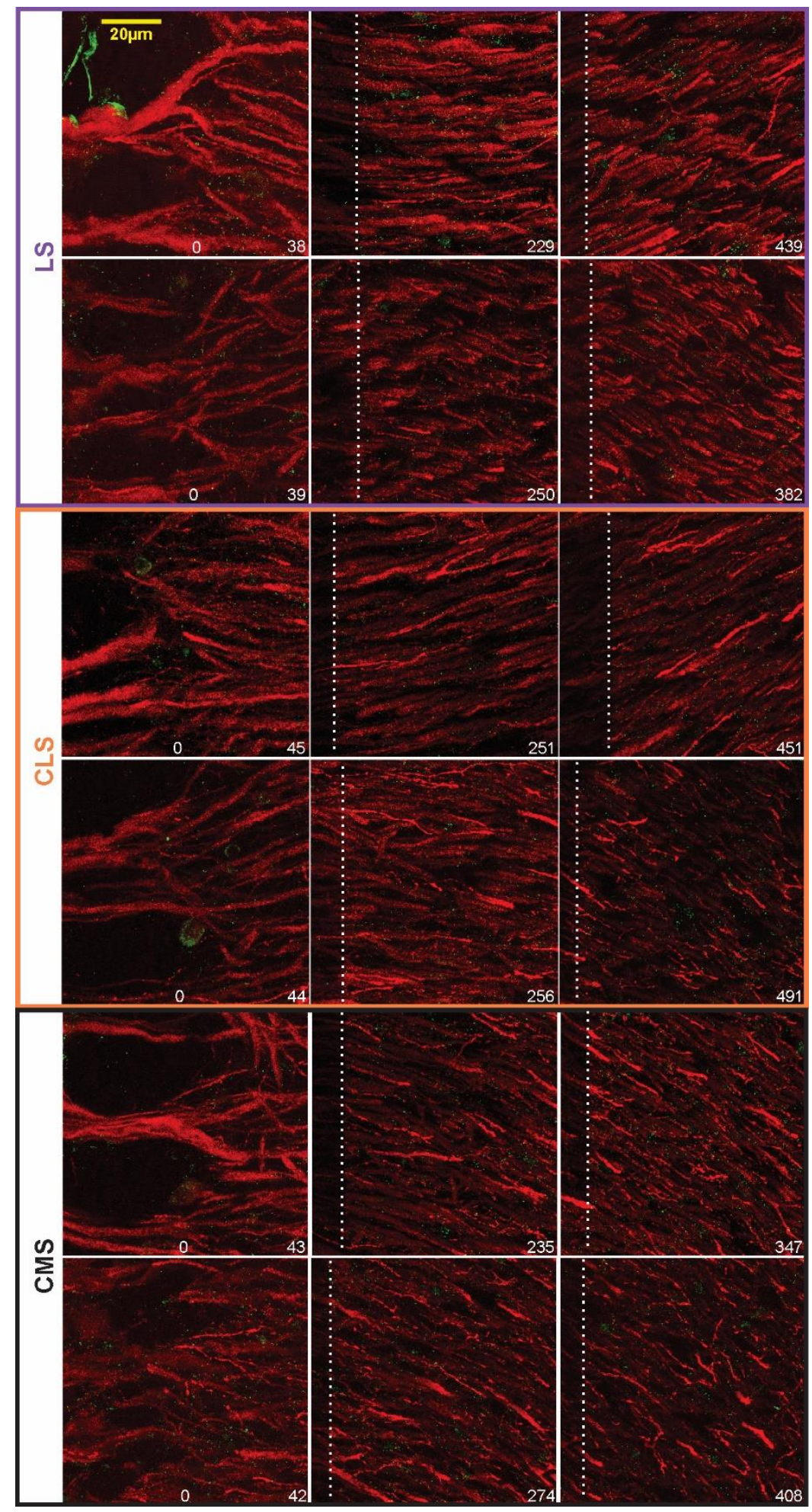

Figure 4: Examples of Nav-Map 2 expression in the ELL. Confocal images from 2 brains (top vs bottom rows for each map) taken at different distances from the tSF. The location of the distal edge of the image is specified in $\mu \mathrm{m}$ at the bottom right of each image. The white dashed lines indicate the edge of the previous -overlapping- scan and thus the pixels on the left of this line are bleached from the previous scan. 


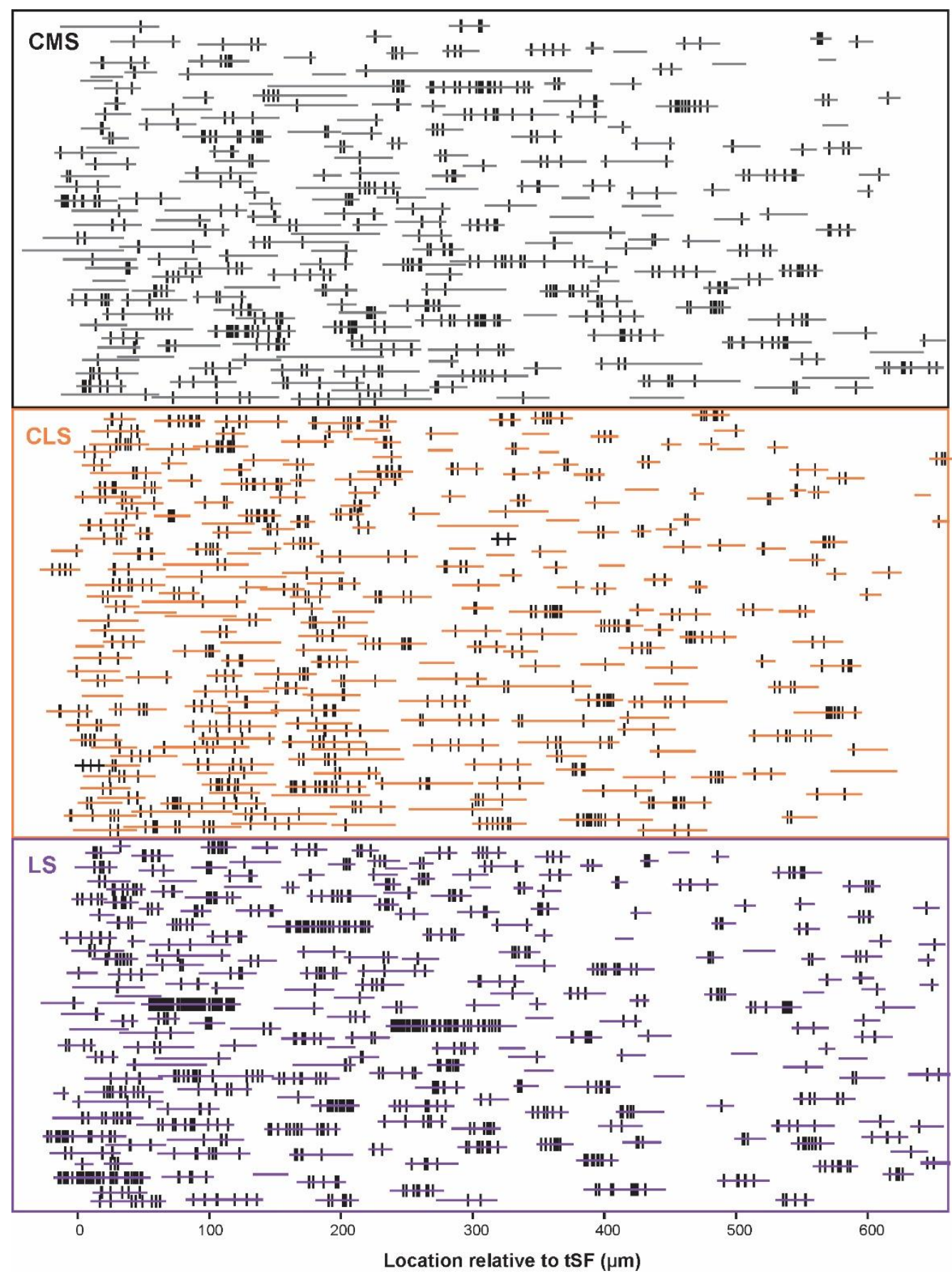

Figure 5: Schematic representation of the entire dataset. The position of each dendrite quantified is depicted by the horizontal colored lines. The position of each Nav puncta identified are marked by the vertical black lines. The vertical stacked arrangement of the dendrites loosely follows the order of processing and thus data from a given slice/brain are found in adjacent rows of the stack. We quantified 727 dendrites in 15 brain slices, totaling $>21400 \mu \mathrm{m}$ of dendrite, and identified 2435 puncta of Nav labelling across segments/locations. 
PCs can be classified in several subcategories (deep, intermediate or superficial PCs and basilar or non-basilar) organized in microcolumns and their apical dendrites can overlap at the same location in the molecular layer. Our method cannot determine which PC neuron a dendrite belongs to and thus we cannot determine to what extent the variability observed is due to variations across -vs. within- subcategories.

The density of puncta per $\mu \mathrm{m}$ of dendrite was calculated for each of the $\sim 250$ dendrites per segment as a sum across all 15 sections and displayed, as a function of dendrite position along the molecular layer, in a 3D histogram (Figure 6A). The pattern of Nav expression clearly differs across segments. In LS, a large proportion of dendrites have a puncta density higher than 0.1 puncta/ $\mu \mathrm{m}$ whereas CMS dendrite have densities mostly below 0.1 puncta/ $\mu \mathrm{m}$. Our data also shows that density stay similar throughout the molecular layer up to -at least- $600 \mu \mathrm{m}$ away for tSF. Our data beyond $600 \mu \mathrm{m}$ are more sparse, nevertheless, the data we do have suggest that more distal dendrites express Nav channels with a density similar to more proximal dendrites (see Figure 5 and 6A). Our data demonstrates that dendrites in the LS have Nav puncta densities about twice as high as CMS dendrites and 1.4 times higher than CLS dendrites (Figure 6B-C). The distribution of Nav puncta along the first $600 \mu \mathrm{m}$ of dendrites (for which we have most data) was not very different across segments. Overall, we see similar densities over the length of the dendrites in LS and CLS but channel density increased slightly in CMS for dendrites located further from tSF (Figure 6D). Nevertheless, our data shows that, despite small differences across segments, we see a qualitatively similar distribution of Nav puncta over the entire length of dendrites considered here $(0-600 \mu \mathrm{m}$ from tSF dorsal edge; Figure 6E).

The differences in Nav channels density across segment could have a significant impact on the neuron's response properties. The impact on neural dynamic and sensory processing is hard to gauge because PCs differ in many aspects between segments. Differences in ion channels composition, tuning, connectivity and more, interacts intricately with the dynamics imposed by Nav channels. Even potential studies using modeling, where a single parameter can be altered (e.g. Nav channels density), presents important challenges since all the elements of the neurons and of the network known to influence the neuron's response dynamic should be included to understand the effect of changing Nav density. Such modelling effort is beyond the scope of this manuscript. 


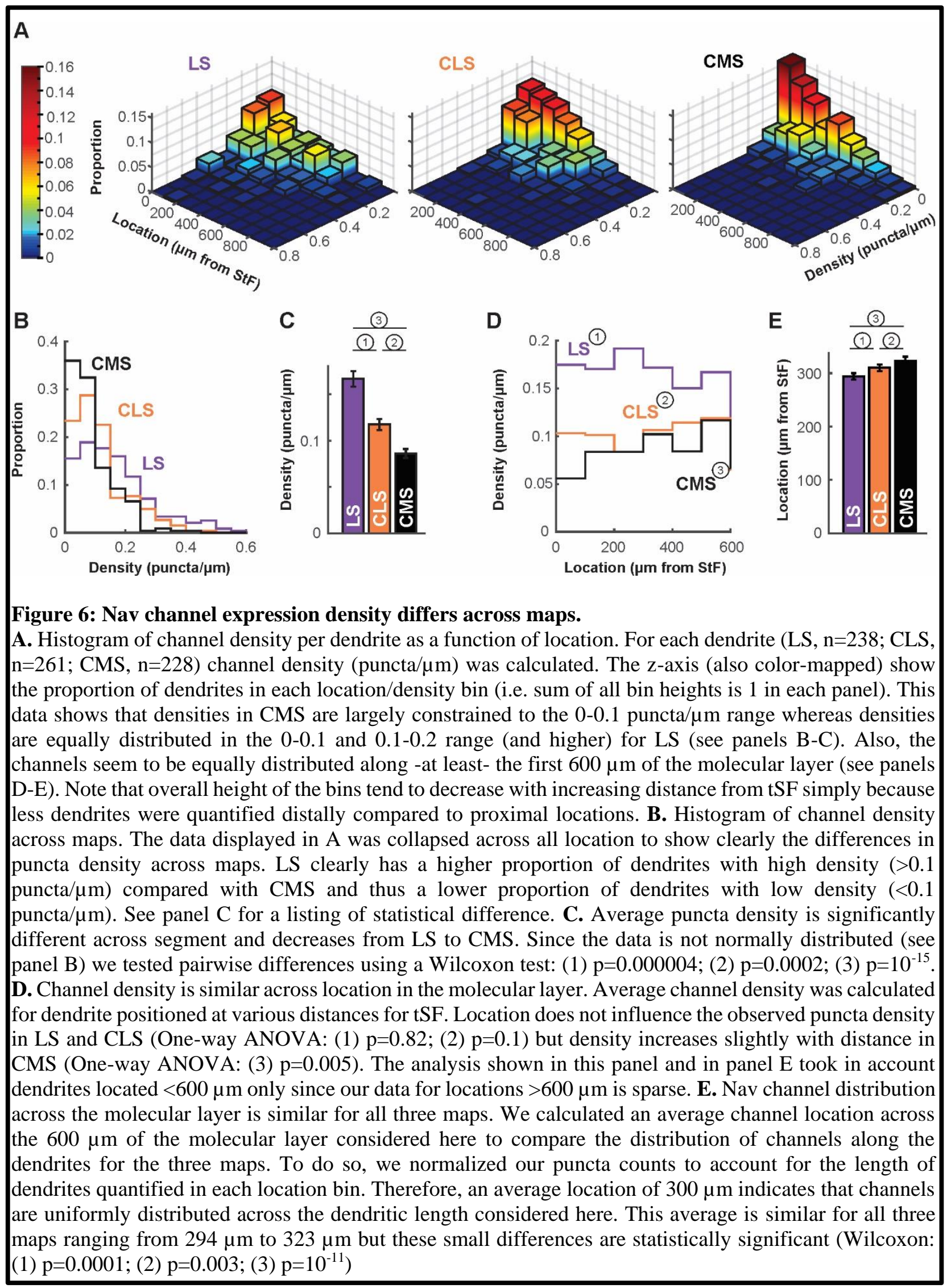



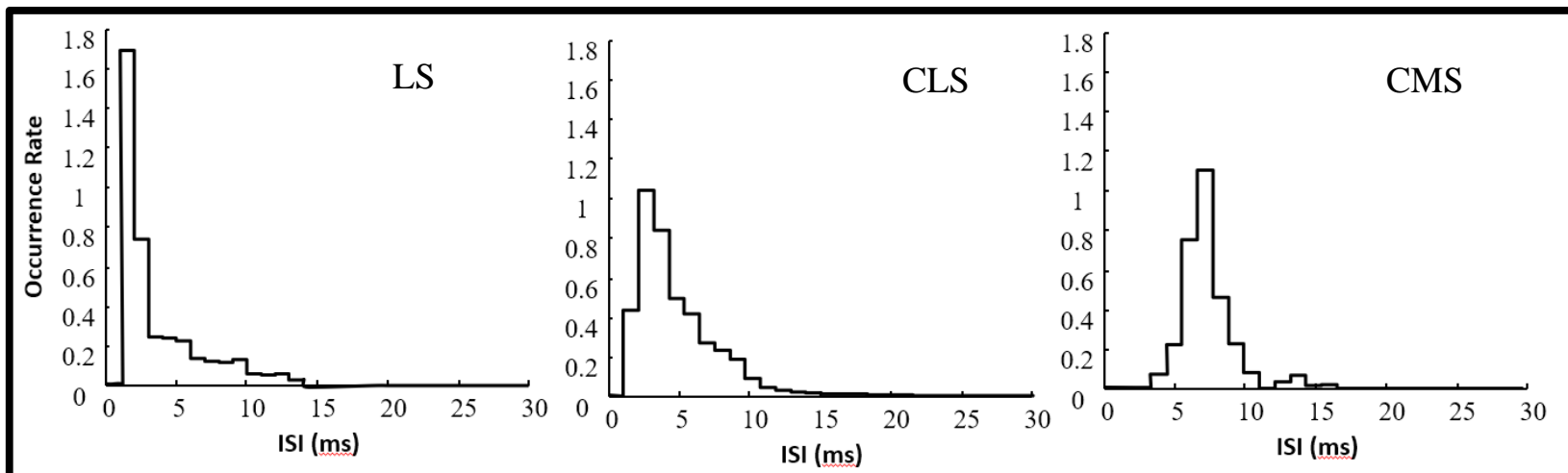

Figure 7: Inter-spike intervals (ISI) histogram for burst-spikes during spontaneous activity. Burst of spikes were identified as described in the methods and the interval between the spikes in each burst contributed to the histograms displayed. The average interval is shorter in LS than CMS with significant differences across all three segments (averages \pm s.e.: $L S=3.2 \pm 0.4$; CLS $=4.6 \pm 0.7$; CMS $=6.7 \pm 0.6$. Wilcoxon test: LS-CLS, $\mathrm{p}<0.00001$; LS-CMS, $\mathrm{p}<10-11$; CLS-CMS, $\mathrm{p}<10-9 . \mathrm{n}=13$ to 19 neurons).

Nevertheless, we suggest that looking at spontaneous activity of the neurons could give us useful insight into the effect of differences in Nav density. Interspike-intervals (ISIs) during spontaneous activity are largely determined by the neuron's intrinsic mechanisms since synaptic inputs are weak and relatively constant. Furthermore, burst ISIs are heavily influenced by only a few ion channels, first and foremost the Nav channels in apical dendrites. Therefore, we investigated potential differences across segments in burst ISIs during spontaneous activity. Focusing on superficial pyramidal cells, we show that burst ISIs tend to be shorter in LS than in CMS (CLS being intermediate; Figure 7, Data obtained from Daniel Williamson). This trend is consistent with a previous observations (Mehaffey et al., 2008b; Turner et al., 1996) and could potentially be attributed in part to the higher density of Nav channels in LS (see discussion).

\section{$\underline{\text { V. Discussion }}$}

\section{Dendritic Nav channel expression and firing patterns}

The principle finding of the present experiment was that the lateral segment of the ELL exhibits the highest dendritic Nav channel density, followed by CLS and CMS. Nav channel densities remain relatively constant along the proximo-distal axes of the apical dendrites across segments with CMS showing slightly higher Nav density in the distal regions.

Previous studies have established the subcellular distribution and function of TTX sensitive Nav channels using immunocytochemistry and electrophysiology techniques (Turner et al., 1994). 
Punctate regions of Nav immunolabel were detected in pyramidal cell somata, basal and apical dendrites. Dendritic Nav channels allow the active propagation of an antidromic spike over around $200 \mu \mathrm{m}$ of proximal apical dendrite, resulting in a DAP at the soma after each somatic spike which underlies the production of burst discharge. Focal ejections of TTX inactivates Nav channels, decreasing spike frequency (Oswald et al., 2004; Turner et al., 1994). Higher conductance from Nav channels does not necessarily lead to higher firing rates. Decreased $\mathrm{Na}^{+}$conductance in dendrites can increase excitability of the soma by delaying the DAP thereby enhancing the socalled "ping-pong" dendro-somatic dynamics that underlies burst generation (Fernandez et al., 2005). There is thus a non-monotonic relationship between $\mathrm{Na}^{+}$conductance and cell excitability (Fernandez et al., 2005) or the amount of information carried by bursts (Doiron et al., 2007) with a maxima at intermediate values. Nevertheless, modelling studies indicate that an increase in

dendritic conductance from Nav channels systematically causes increased burst rates (Doiron et al., 2007).

Backpropagation has been characterized as traveling up $200 \mu \mathrm{m}$ before the active propagation is not detected. Our results, and the original study that identified the Nav channel's presence in the dendrites (Turner et al., 1994), show that the channels are distributed along all -or most- of the dendritic tree. The role of these channels, past the $200 \mu \mathrm{m}$ where backpropagation is evident, is unknown. One possibility is that they contribute to the DAP but the current they generate in the more distal dendrites is too small to be clearly identified. Another possibility involves an interaction between the channels and synaptic inputs. GABAergic inputs have been shown to influence the DAP generation (Mehaffey et al., 2005), it is therefore not unlikely that the $\mathrm{Na}+$ current in the distal dendrites shapes and affects the dynamics of synaptic inputs. Given the important role of feedback inputs onto these apical dendrites the presence of the channels near these synapses could alter the neuron's response properties significantly.

\section{Segment-specific regulation of bursting mechanisms}

The effect of variations in the DAP current are particularly hard to predict because several other currents overlap with the depolarization from dendritic Nav channels. Kv3.3 channels, muscarinic or 5HT receptors, $M$ or A current and GABAergic inputs can influence the afterpotential (Marquez et al., 2013). In particular, somatic spikes are followed by both fast and slow AHPs that help repolarize the cell and lengthen the interval to the next spikes (Turner et al., 2002). 
Therefore, the DAP and the AHP have opposite influences on bursting. ELL expresses 2 different subtypes of SK channels (SK1: dendrites; SK2: soma) that cause AHPs (Ellis et al., 2007b, 2008) following a gradient where LS has a denser distribution than CMS. The relatively short DAP (8$10 \mathrm{~ms}$ ) temporally overlaps with the longer AHP and their strength varies with cell subtype. It is possible that higher expression of dendritic Nav channels may partially compensate for higher hyperpolarizing currents and allow these currents to be modulated with different gains.

Several mechanisms for external modulation of these hyperpolarizing and depolarizing currents have been characterized. Inhibitory interneurons are prevalent in the ELL (e.g. VML cells or granular cells) and synapse both on PC's soma and dendritic arbor (Berman and Maler, 1999). Dendritic application of GABAA agonist can affect the dendritic leak conductance leading to a DAP reduction and thus have a divisive effect on the cell's input-output relationship (Mehaffey et al., 2005). In contrast, somatic inhibition has a subtractive effect in suprathreshold regime and divisive in subthreshold regime since the reversal potential of $\mathrm{GABA}_{\mathrm{A}}$ channels is close to the neuron's resting potential (Doiron et al., 2002). Therefore, somatic inhibition could also potential interact with the subthreshold dynamic influenced by the DAP and AHPs. Since somatic inhibition varies across segments (Hofmann and Chacron, 2017, Allen and Marsat, unpublished observation), GABAergic inhibition is another segment-specific factor that could interact with the current from dendritic Nav channels.

5-HT is an important modulator of social behavior that is released during communication and LS shows the highest 5-HT innervation (Johnston et al., 1990). Interestingly, the innervation pattern is also layer specific across segments, where LS shows dense expression in PCL and VML. 5-HT increases burst firing across segments with the greatest effect in the LS which is consistent with its expression density (Deemyad et al., 2011). These effects are mediated by 5-HT2 receptors (Larson et al., 2014) that increase PC excitability and bursting via downregulation of SK channel and M-type potassium channel currents that contribute to the AHP (Deemyad et al., 2013).

Given that several factors affect the shape of spike's after-potential and the bursting dynamic we cannot be certain that the shorter burst ISIs we observed in LS are due to the denser Nav expression but it is a plausible factor. A strong DAP, that peaks a few ms after the spike, could explain the relatively high probability of having 2-4 ms ISIs in LS whereas the longer-lasting AHPs strongly influencing LS ON-cells could lead to the relatively low probability of ISIs longer than 5ms. The longer burst ISIs observed in CMS could result from the lower Nav current since it can 
delay the DAP (Fernandez et al., 2005) and is not opposed by a strong AHP in superficial PCs. We therefore propose that differences in Nav channel expression interacts with other aspect of the neuron's dynamic to influence the spiking patterns.

\section{Bursts and neural coding}

Bursts have a well-defined role in coding for specific temporal features of sensory signals. The relationship between patterns of spikes within a burst can further signal details about the feature that triggered the burst (Oswald et al., 2007). Specifically, the ISI within the burst is correlated with the amplitude and slope of the upstroke it signals. Studies in several sensory systems have shown that burst structure can carry information about the stimulus (Guido et al., 1995; Lesica and Stanley, 2004) and that this information is behaviorally relevant (Marsat and Pollack, 2010). Our results point to the possibility that variations in Nav channels may influence the burst dynamic to adjust the correlation between burst ISI and stimulus features. By adjusting the range of ISI coding for specific portions of stimulus space, each ELL segment could have its burst-code adjusted for slightly different stimulus features. We already know that bursts are involved in processing different signals across segments since bursts signal the presence of certain types of chirps in LS but not the other segments (Marsat et al., 2009). Also, bursts in CLS and CMS might be more specifically dedicated to signaling prey-like stimuli (Nelson and Maciver, 1999). Differences in Nav expression could thus contribute to these different roles of burst coding across segments.

We started this chapter by pointing out two perspectives on neural heterogeneity. One highlights how similar neural outputs can arise from diverse combinations of neural properties. The other focuses on the need to vary neural physiology in order to adjust and specialize cells for different purposes. Our study does not determine what role the variability in dendritic Nav channel expression plays: compensating for other neural properties to keep burst coding functioning similarly across segments or on the contrary adjusting burst coding for specific roles. Since the two possibilities are not exclusive, we speculate that the difference we describe in our study could partly compensate for other variations in PCs properties while also influencing burst coding to improve the ability of the different segments to perform specific tasks. 


\section{CHAPTER 3: CONCLUSION AND FUTURE DIRECTIONS}

In the present study, we used immunohistochemical labeling and quantification to investigate the spatial distribution of Nav channels across the three electrosensory maps of the ELL of the fish Apteronotus leptorhynchus. We also investigated whether Nav channel density varies based on location of the dendrite from the pyramidal cell layer. We demonstrate that dendritic Nav channel expression in the ELL of A leptorhynchus exhibits a mediolateral gradient with lateral segment exhibiting the highest density (Fig 6B). Dendrites quantified in the LS show twice as high Nav puncta when compared to CMS, and 1.4 times as high as observed in CLS dendrites (Fig 6C). It suggests a potential role for this differential dendritic Nav channel expression in counteracting other ionic conductances at play (for e.g., SK, Kv3.3; Marquez et al., 2013). However, since ON- and OFF-cells within each map are grouped together during Nav channel quantification in this study, it is unknown if the differences in Nav channel distribution patterns seen across maps hold true for both ON- and OFF-cells separately within each map. Therefore, we do not know if the mediolateral gradient seen in the dendritic Nav expression pattern arises from both cell types or is contributed by one of the two. There are no dendritic molecular markers which are specific to either of the cell types that would allow us to distinguish them apart. However, iontophoretic intracellular dye injections in single neurons coupled with immunohistochemistry (Lichtman and Conchello, 2005) can be used to further delineate dendritic Nav expression pattern between $\mathrm{ON}$ - and OFF-cells within maps.

TTX-sensitive Nav channels are shown to support active backpropagation that underlies burst generation in this system (Turner et al., 1994). We presented a first step in clarifying the effect of Nav density on bursting and burst-coding by showing that neurons of different segments have different burst ISIs. We argue that this difference in burst ISIs could be due -at least partlyto the difference in Nav channel density and that it could impact the burst coding scheme, particularly the way burst ISI correlate with specific aspects of the stimulus. A full study of burst coding across segments could reveal interesting difference relatable to differences in $\mathrm{Na}^{+}$currents. One complication however is that several factors can affect bursting and these factors are sometimes correlated with the differences in Nav channel expression. Therefore, it would be difficult to assign a causative relationship to any correlation we observe between Nav expression and burst coding. A more targeted approach would consist of filling with dye individual neurons 
after they are probed for neural coding properties and assess their Nav expression with immunolabelling. By characterizing the correlation between Nav expression and coding in individual neurons of the same sub-type, we minimize the risk that another factor that co-varies across segments with Nav expression would be the cause of the observed correlation. Nevertheless, this approach can still not resolve a cause-effect relationship from a simple correlation. A useful complementary strategy to address this issue would be to use modelling. Detailed models of PCs already exist and could be supplemented/altered as necessary. The main difficulty with this approach is that it would require incorporating all the factors that vary across segments to account for the impact of Nav density. Nav density could then be altered in the model as the only independent variable and the impact on coding characterized.

Ion channel composition of PCs is one of the factors that dictates the bursting patterns observed. In addition to Nav and SK channels, ELL of A. leptorhynchus expresses a wide range of other ion channels with varied kinetics and in different gradients across maps (Marquez et al., 2013). One of them is the high-threshold potassium channel- Kv3.3 that is shown to regulate burst discharge and enable high frequency firing in PCs by decreasing accumulation of low-threshold potassium currents (Mehaffey et al., 2006). Dense Kv3.3 expression is seen in PCL, GCL and DNL with lower density expression in molecular layer across ELL maps. PCs present in the deep, intermediate and superficial layers, all show Kv3.3 expression along the soma and both proximal and distal dendrites (Mehaffey et al., 2006; Rashid et al., 2001b). These TEA sensitive channels display a slow rate of inactivation in both somatic and dendritic compartments of PCs and play a role in repolarizing both backpropagating dendritic and somatic spikes (Rashid et al., 2001a, 2001b). Since they differentially regulate burst output of the cell by regulating the extent of somatic re-excitation by backpropagating dendritic $\mathrm{Na}^{+}$spikes (Noonan et al., 2003), determining the interplay of these currents with DAPs of different sizes could allow us to better understand the dynamic of burst generation and its regulation across segments.

One of the principles of dendritic excitability is the non-uniform expression of voltage gated channels that likely have functional implication. For instance, Kv4 channel expression in CA1 PCs shows $\sim 70 \%$ increase in density along the proximo-distal axis of apical dendrites (Kerti et al., 2012). In chapter 2, we show that dendritic Nav show a slight increase in their density on distal apical dendrites in CMS and no such increase in Nav density was observed along the proximo-distal axis for LS and CLS. As ON and OFF-cells within a map are pooled in this study, 
we do not know if this expression is specific to one or constant across cell types in CMS. This difference could be further investigated by using iontophoretic lucifer yellow injections coupled with immunohistochemistry (Lichtman and Conchello, 2005).

Furthermore, the role of Nav channels in the more distal parts of the apical dendrites is unknown. Active backpropagation mostly occurs in the $200 \mu \mathrm{m}$ most proximal to the soma and it is unclear whether more distal Nav channel can contribute to the DAP. Both proximal and apical dendrites receive feedback inputs and inputs from inhibitory interneurons (Maler, 2007). We know that synaptic current can interact with the DAP generation through an effect on leak conductances (Mehaffey et al., 2005) or due to the shunting nature of $\mathrm{GABA}_{\mathrm{A}}$ inhibition (Doiron et al., 2002). Therefore, we suspect that Nav channels can shape synaptic inputs and thus influence the function of feedback inputs. Electrophysiological or functional imaging of dendrites could start to address this hypothesis.

To conclude, in this study, we have investigated dendritic Nav expression density in pyramidal cell's apical dendrites and showed they differ across ELL segments. By shaping the bursting dynamic, these differences could fine tune the coding properties of each segment to better match their role in electrosensory processing. Our results can thus help to link specific molecular mechanisms to sensory processing and perception. 


\section{REFERENCES}

Ban, Y., Smith, B. E., and Markham, M. R. (2015). A highly polarized excitable cell separates sodium channels from sodium-activated potassium channels by more than a millimeter. $J$. Neurophysiol. 114, 520-530. doi:10.1152/jn.00475.2014.

Bastian, J., Chacron, M. J., and Maler, L. (2004). Plastic and Nonplastic Pyramidal Cells Perform Unique Roles in a Network Capable of Adaptive Redundancy Reduction. Neuron 41, 767-779. doi:10.1016/s0896-6273(04)00071-6.

Bastian, J., and Courtright, J. (1991). Morphological correlates of pyramidal cell adaptation rate in the electrosensory lateral line lobe of weakly electric fish. J. Comp. Physiol. A 168, 393 407. doi:10.1007/bf00199600.

Bastian, J., and Nguyenkim, J. (2001). Dendritic Modulation of Burst-Like Firing in Sensory Neurons. J. Neurophysiol. 85, 10-22. doi:10.1152/jn.2001.85.1.10.

Berman, N. J., and Maler, L. (1999). Neural architecture of the ELL.

Cattaneo, A., Maffei, L., and Morrone, C. (1981). Two firing patterns in the discharge of complex cells encoding different attributes of the visual stimulus. Exp. Brain Res. 43, 115118. doi:10.1007/bf00238819.

Deemyad, T., Maler, L., and Chacron, M. J. (2011). Inhibition of SK and M channel-mediated currents by 5 -HT enables parallel processing by bursts and isolated spikes. J. Neurophysiol. 105, 1276-1294. doi:10.1152/jn.00792.2010.

Deemyad, T., Metzen, M. G., Pan, Y., and Chacron, M. J. (2013). Serotonin selectively enhances perception and sensory neural responses to stimuli generated by same-sex conspecifics. Proc. Natl. Acad. Sci. 110, 19609-19614. doi:10.1073/pnas.1314008110.

Deng, Q. (2005). A C-Terminal Domain Directs Kv3.3 Channels to Dendrites. J. Neurosci. 25, 11531-11541. doi:10.1523/jneurosci.3672-05.2005.

Doiron, B., Laing, C., Longtin, A., and Maler, L. (2002). Ghostbursting: a novel neuronal burst mechanism. J. Comput. Neurosci. 12, 5-25.

Doiron, B., Oswald, A.-M. M., and Maler, L. (2007). Interval Coding. II. Dendrite-Dependent Mechanisms. J. Neurophysiol. 97, 2744-2757. doi:10.1152/jn.00988.2006.

Duménieu, M., Oulé, M., Kreutz, M. R., and Lopez-Rojas, J. (2017). The Segregated Expression of Voltage-Gated Potassium and Sodium Channels in Neuronal Membranes: Functional Implications and Regulatory Mechanisms. Front. Cell. Neurosci. 11, 115. doi:10.3389/fncel.2017.00115.

Ellis, L. D., Krahe, R., Bourque, C. W., Dunn, R. J., and Chacron, M. J. (2007a). Muscarinic 
Receptors Control Frequency Tuning Through the Downregulation of an A-Type Potassium Current. J. Neurophysiol. 98, 1526-1537. doi:10.1152/jn.00564.2007.

Ellis, L. D., Maler, L., and Dunn, R. J. (2008). Differential distribution of SK channel subtypes in the brain of the weakly electric fish Apteronotus leptorhynchus. J. Comp. Neurol. 507, 1964-1978. doi:10.1002/cne.21597.

Ellis, L. D., Mehaffey, W. H., Harvey-Girard, E., Turner, R. W., Maler, L., and Dunn, R. J. (2007b). SK Channels Provide a Novel Mechanism for the Control of Frequency Tuning in Electrosensory Neurons. J. Neurosci. 27, 9491-9502. doi:10.1523/jneurosci.1106-07.2007.

Fernandez, F. R., Mehaffey, W. H., and Turner, R. W. (2005). Dendritic Na + Current Inactivation Can Increase Cell Excitability By Delaying a Somatic Depolarizing Afterpotential. J. Neurophysiol. 94, 3836-3848. doi:10.1152/jn.00653.2005.

Gabbiani, F., Metzner, W., Wessel, R., and Koch, C. (1996). From stimulus encoding to feature extraction in weakly electric fish. Nature 384, 564-567. doi:10.1038/384564a0.

Guido, W., Lu, S. M., and Sherman, S. M. (1992). Relative contributions of burst and tonic responses to the receptive field properties of lateral geniculate neurons in the cat. $J$. Neurophysiol. 68, 2199-2211. doi:10.1152/jn.1992.68.6.2199.

Guido, W., Lu, S. M., Vaughan, J. W., Godwin, D. W., and Sherman, S. M. (1995). Receiver operating characteristic (ROC) analysis of neurons in the cat's lateral geniculate nucleus during tonic and burst response mode. Vis. Neurosci. 12, 723-741. doi:10.1017/s0952523800008993.

Heiligenberg, W., and Dye, J. (1982). Labelling of electroreceptive afferents in a gymnotoid fish by intracellular injection of HRP: The mystery of multiple maps. J. Comp. Physiol. - A Sensory, Neural, Behav. Physiol. 148, 287-296. doi:10.1007/bf00679013.

Hille, B. (2001). Ion channels of excitable membranes. 3rd ed. Sunderland, Massachusetts, USA: Sinauer Associates.

Hofmann, V., and Chacron, M. J. (2017). Differential receptive field organizations give rise to nearly identical neural correlations across three parallel sensory maps in weakly electric fish. doi:10.1371/journal.pcbi.1005716.

Huerta, P. T., and Lisman, J. E. (1995). Bidirectional synaptic plasticity induced by a single burst during cholinergic theta oscillation in CA1 in vitro. Neuron 15, 1053-1063. doi:10.1016/0896-6273(95)90094-2.

Izhikevich, E. (2006). Bursting. Scholarpedia 1, 1300. doi:10.4249/scholarpedia.1300.

Johnston, S. A., Maler, L., and Tinner, B. (1990). The distribution of serotonin in the brain of Apteronotus leptorhynchus: an immunohistochemical study. J. Chem. Neuroanat. 3, 42965. 
Kawasaki, M. (2005). "Physiology of Tuberous Electrosensory Systems," in Electroreception, eds. T. H. Bullock, C. D. Hopkins, A. N. Popper, and R. R. Fay (Springer New York), 154194. doi:10.1007/0-387-28275-0_7.

Kepecs, A., Wang, X.-J., and Lisman, J. (2002). Bursting neurons signal input slope. J. Neurosci. 22, 9053-62. doi:10.1523/jneurosci.22-20-09053.2002.

Kerti, K., Lorincz, A., and Nusser, Z. (2012). Unique somato-dendritic distribution pattern of Kv4.2 channels on hippocampal CA1 pyramidal cells. Eur. J. Neurosci. 35, 66-75. doi:10.1111/j.1460-9568.2011.07907.x.

Krahe, R., Bastian, J., and Chacron, M. J. (2008). Temporal Processing Across Multiple Topographic Maps in the Electrosensory System. J. Neurophysiol. 100, 852-867. doi:10.1152/jn.90300.2008.

Krahe, R., and Gabbiani, F. (2004). Burst firing in sensory systems. Nat. Rev. Neurosci. 5, 1323. doi:10.1038/nrn1296.

Krahe, R., and Maler, L. (2014). Neural maps in the electrosensory system of weakly electric fish. Curr. Opin. Neurobiol. 24, 13-21. doi:10.1016/j.conb.2013.08.013.

Larson, E. A., Metzen, M. G., and Chacron, M. J. (2014). Serotonin modulates electrosensory processing and behavior via 5-HT2-like receptors. Neuroscience 271, 108-118. doi:10.1016/j.neuroscience.2014.04.033.

Lemon, N., and Turner, R. W. (2000). Conditional Spike Backpropagation Generates Burst Discharge in a Sensory Neuron. J. Neurophysiol. 84, 1519-1530. doi:10.1152/jn.2000.84.3.1519.

Lesica, N. A., and Stanley, G. B. (2004). Encoding of Natural Scene Movies by Tonic and Burst Spikes in the Lateral Geniculate Nucleus. J. Neurosci. 24, 10731-10740. doi:10.1523/jneurosci.3059-04.2004.

Lesica, N. A., Weng, C., Jin, J., Yeh, C.-I., Alonso, J.-M., and Stanley, G. B. (2006). Dynamic Encoding of Natural Luminance Sequences by LGN Bursts. PLoS Biol. 4, e209. doi:10.1371/journal.pbio.0040209.

Lichtman, J. W., and Conchello, J.-A. (2005). Fluorescence microscopy. Nat. Methods 2, 910919. doi:10.1038/nmeth817.

Lisman, J. (1997). Bursts as a unit of neural information: making unreliable synapses reliable. Trends Neurosci. 20, 38-43. doi:10.1016/s0166-2236(96)10070-9.

Llináis, R. R. (2014). Intrinsic electrical properties of mammalian neurons and CNS function: a historical perspective. Front. Cell. Neurosci. 8, 1-14. doi:10.3389/fncel.2014.00320.

Maler, L. (1979). The posterior lateral line lobe of certain gymnotoid fish: Quantitative light 
microscopy. J. Comp. Neurol. 183, 323-363. doi:10.1002/cne.901830208.

Maler, L. (2007). "Neural strategies for optimal processing of sensory signals," in Progress in Brain Research, 135-154. doi:10.1016/s0079-6123(06)65009-7.

Maler, L. (2009). Receptive field organization across multiple electrosensory maps. I. Columnar organization and estimation of receptive field size. J. Comp. Neurol. 516, 376-393. doi: $10.1002 /$ cne.22124.

Marquez, B. T., Krahe, R., and Chacron, M. J. (2013). Neuromodulation of early electrosensory processing in gymnotiform weakly electric fish. J. Exp. Biol. 216, 2442-2450. doi:10.1242/jeb.082370.

Marsat, G., and Pollack, G. S. (2006). A Behavioral Role for Feature Detection by Sensory Bursts. J. Neurosci. 26, 10542-10547. doi:10.1523/jneurosci.2221-06.2006.

Marsat, G., and Pollack, G. S. G. S. (2010). The structure and size of sensory bursts encode stimulus information but only size affects behavior. J. Comp. Physiol. A. 196, 315-320. doi:10.1007/s00359-010-0514-8.

Marsat, G., and Pollack, G. S. G. S. (2012). Bursting Neurons and Ultrasound Avoidance in Crickets. Front. Neurosci. 6, 95. doi:10.3389/fnins.2012.00095.

Marsat, G., Proville, R. D., and Maler, L. (2009). Transient Signals Trigger Synchronous Bursts in an Identified Population of Neurons. J. Neurophysiol. 102, 714-723. doi:10.1152/jn.91366.2008.

Mehaffey, W. H., Doiron, B., Maler, L., and Turner, R. W. (2005). Deterministic Multiplicative Gain Control with Active Dendrites. J. Neurosci. 25, 9968-9977. doi:10.1523/jneurosci.2682-05.2005.

Mehaffey, W. H., Ellis, L. D., Krahe, R., Dunn, R. J., and Chacron, M. J. (2008a). Ionic and neuromodulatory regulation of burst discharge controls frequency tuning. J. Physiol. - Paris 102, 195-208. doi:10.1016/j.jphysparis.2008.10.019.

Mehaffey, W. H., Fernandez, F. R., Rashid, A. J., Dunn, R. J., and Turner, R. W. (2006). Distribution and function of potassium channels in the electrosensory lateral line lobe of weakly electric apteronotid fish. J. Comp. Physiol. A Neuroethol. Sensory, Neural, Behav. Physiol. 192, 637-648. doi:10.1007/s00359-006-0103-z.

Mehaffey, W. H., Maler, L., and Turner, R. W. (2008b). Intrinsic Frequency Tuning in ELL Pyramidal Cells Varies Across Electrosensory Maps. J. Neurophysiol. 99, 2641-2655. doi:10.1152/jn.00028.2008.

Metzner, W., and Juranek, J. (1997). A sensory brain map for each behavior? Proc. Natl. Acad. Sci. U. S. A. 94, 14798-14803. doi:10.1073/pnas.94.26.14798. 
Metzner, W., Koch, C., Wessel, R., and Gabbiani, F. (1998). Feature Extraction by Burst-Like Spike Patterns in Multiple Sensory Maps. J. Neurosci. 18, 2283-2300.

doi:10.1523/jneurosci.18-06-02283.1998.

Mukherjee, P., and Kaplan, E. (1995). Dynamics of neurons in the cat lateral geniculate nucleus: in vivo electrophysiology and computational modeling. J. Neurophysiol. 74, 1222-1243. doi:10.1152/jn.1995.74.3.1222.

Nelson, M. E., and Maciver, M. A. (1999). Prey capture in the weakly electric fish Apteronotus albifrons: sensory acquisition strategies and electrosensory consequences. J. Exp. Biol. 202, 1195-203.

Nelson, M. E., Xu, Z., and Payne, J. R. (1997). Characterization and modeling of P-type electrosensory afferent responses to amplitude modulations in a wave-type electric fish. $J$. Comp. Physiol. A. 181, 532-44.

Noonan, L., Doiron, B., Laing, C., Longtin, A., and Turner, R. W. (2003). A Dynamic Dendritic Refractory Period Regulates Burst Discharge in the Electrosensory Lobe of Weakly Electric Fish. J. Neurosci. 23, 1524-1534. doi:10.1523/jneurosci.23-04-01524.2003.

Olesens, O. F. (1994). Expression of Low Molecular Weight Isoforms of Microtubule-associated Protein 2. J. Biol. Chem. 269, 32904-32908.

Oswald, A.-M. M., Chacron, M. J., Doiron, B., Bastian, J., and Maler, L. (2004). Parallel Processing of Sensory Input by Bursts and Isolated Spikes. J. Neurosci. 24, 4351-4362. doi:10.1523/jneurosci.0459-04.2004.

Oswald, A.-M. M., Doiron, B., and Maler, L. (2007). Interval Coding. I. Burst Interspike Intervals as Indicators of Stimulus Intensity. J. Neurophysiol. 97, 2731-2743. doi:10.1152/jn.00987.2006.

Prinz, A. A., Bucher, D., and Marder, E. (2004). Similar network activity from disparate circuit parameters. Nat. Neurosci. 7, 1345-1352. doi:10.1038/nn1352.

Rashid, A. J., Dunn, R. J., and Turner, R. W. (2001a). A prominent soma-dendritic distribution of Kv3.3 K+ channels in electrosensory and cerebellar neurons. J. Comp. Neurol. 441, 234 247. doi:10.1002/cne.1409.

Rashid, A. J., Morales, E., Turner, R. W., and Dunn, R. J. (2001b). The Contribution of Dendritic Kv3 K + Channels to Burst Threshold in a Sensory Neuron. J. Neurosci. 21, 125135. doi:10.1523/jneurosci.21-01-00125.2001.

Shumway, C. (1989). Multiple electrosensory maps in the medulla of weakly electric gymnotiform fish. I. Physiological differences. J. Neurosci. 9, 4388-4399. doi:10.1523/jneurosci.09-12-04388.1989.

Stuart, G. J., and Sakmann, B. (1994). Active propagation of somatic action potentials into 
neocortical pyramidal cell dendrites. Nature 367, 69-72. doi:10.1038/367069a0.

Turner, R., Maler, L., Deerinck, T., Levinson, S., and Ellisman, M. (1994). TTX-sensitive dendritic sodium channels underlie oscillatory discharge in a vertebrate sensory neuron. $J$. Neurosci. 14, 6453-6471. doi:10.1523/jneurosci.14-11-06453.1994.

Turner, R., Meyers, D., Richardson, T., and Barker, J. (1991). The site for initiation of action potential discharge over the somatodendritic axis of rat hippocampal CA1 pyramidal neurons. J. Neurosci. 11, 2270-2280. doi:10.1523/jneurosci.11-07-02270.1991.

Turner, R. W., Lemon, N., Doiron, B., Rashid, A. J., Morales, E., Longtin, A., et al. (2002). Oscillatory burst discharge generated through conditional backpropagation of dendritic spikes. J. Physiol. 96, 517-530. doi:10.1016/s0928-4257(03)00007-x.

Turner, R. W., Plant, J. R., and Maler, L. (1996). Oscillatory and burst discharge across electrosensory topographic maps. J. Neurophysiol. 76, 2364-2382. doi:10.1152/jn.1996.76.4.2364. 